\title{
Il Modello di Basilea per l'analisi di también: uno studio contrastivo con l'italiano
}

\author{
Giorgia Esposito ${ }^{1}$
}

Ricevuto: 28 febbraio 2019 / Modificato: 21 novembre 2019 / Accettato: 3 febbraio 2020

\begin{abstract}
Riassunto. L'obiettivo di questo studio consiste in un'analisi contrastiva della particella additiva dello spagnolo también e dei suoi possibili traducenti in italiano, con lo scopo di individuare i livelli di analisi più rilevanti in un'ottica contrastiva fra le due lingue. Lo studio è realizzato adottando una prospettiva funzionale e un approccio semasiologico. Inoltre, l'analisi si avvale di un modello di segmentazione pragmatica del testo: il Modello di Basilea (Ferrari et al. 2008), del quale studi precedenti (p. es., Borreguero 2014 e De Cesare / Borreguero 2014) hanno dimostrato la produttività in quanto strumento euristico capace di spiegare il comportamento testuale delle particelle polifunzionali, anche in un'ottica interlinguistica. Ad esempio, la saturazione del Quadro Informativo da parte di también attiva la funzione di connettivo testuale ( $v s$. avverbio focalizzante) che, in un'ottica contrastiva con l'italiano, si traduce nella selezione di equivalenti diversi da anche. Infine, si focalizzerà l'attenzione su alcuni casi in cui l'ambiguità referenziale innescata dall'ampia mobilità distribuzionale di también deve essere necessariamente risolta per veicolare, nella traduzione, il medesimo insieme di elementi in gioco.
\end{abstract}

Parole chiave: Modello di Basilea; particelle discorsive; también; focus; analisi contrastiva spagnoloitaliano.

\section{[en] The Basel Model for the analysis of Spanish también: A contrastive study with Italian}

\begin{abstract}
The present paper aims at performing a contrastive analysis of the Spanish additive particle también and its acceptable equivalents in Italian so as to detect the most relevant levels of cross-linguistic confrontation. A functional perspective and a semasiological approach - i.e. from sign to function - are endorsed for the analysis of the lexeme. Moreover, the study implements a model for paragraph segmentation, namely the Basel Model (Ferrari et al. 2008). According to previous studies (e.g. Borreguero 2014 and De Cesare / Borreguero 2014), this pragmatic segmentation model proves useful in disclosing the textual behavior of polyfunctional markers, even from a contrastive perspective. As a case in point, the collocation of también within the Frame unit entails the activation of the connective ( $v s$. focus adverb) function, which leads to the selection of Italian equivalents that are different from anche. Finally, special attention will be paid to a number of cases related to the referential ambiguity of también, which is prompted by its notable distributional mobility and which ought to be solved in translation in order to convey a matching set of elements in the two versions.
\end{abstract}

Keywords: Basel Model; discourse Particles; también; focus; contrastive analysis Spanish-Italian.

Università degli Studi di Torino. Dipartimento di Lingue e Letterature Straniere e Culture Moderne.

E-mail: giorgia.esposito@unito.it 
Sommario: 1. Introduzione 2. Le particelle additive in spagnolo e in italiano 2.1 También vs. anche 3. Il Modello di Basilea per la segmentazione pragmatica del testo 4. Il MB per l'analisi contrastiva delle particelle additive 4.1 También nelle UI 4.2 También con fuoco a sinistra 5. Conclusioni.

Come citare: Esposito, Giorgia (2020): «Il Modello di Basilea per l'analisi di también: uno studio contrastivo con l'italiano», Cuadernos de Filología Italiana, 27, pp. 77-107

\section{Introduzione}

In questo studio mi propongo di effettuare un'analisi contrastiva spagnolo-italiano per gettare luce sui valori, discussi in letteratura, dell'avverbio focalizzante también e dei suoi possibili traducenti in italiano, attraverso l'impalcatura teorica del Modello di Basilea (MB) (Ferrari et al. 2008). La fonte linguistica da cui estrarrò le occorrenze consiste in un corpus parallelo (Baker 1995: 230), vale a dire, formato da una coppia di testi - o «bi-texts» (Hartmann 2004) - in cui uno è la traduzione dell'altro. Più precisamente, mi baserò su un testo letterario in lingua spagnola: El mal de Montano (Vila-Matas 2002) e sulla sua traduzione in italiano: Il mal di Montano (Vila-Matas 2005). La scelta del corpus risponde fondamentalmente a tre criteri: (i) sincronico, poiché è un testo pubblicato e tradotto negli ultimi vent'anni; (ii) qualitativo, dato che, trattandosi di un testo letterario, permette di contare su una traduzione qualitativamente accurata; (iii) di varietà della lingua, dal momento che, pur trattandosi di un testo letterario, reca maggiormente i tratti del testo argomentativo; infatti, in Vila-Matas (2002) il contesto finzionale serve soprattutto da cornice (o pretesto) per realizzare una critica letteraria non sistematica e per portare avanti un dialogo eterodosso con il canone (Esposito 2019: 251).

Mediante la segmentazione dell'enunciato proposta dal MB, identificherò le correlazioni esistenti fra l'occorrenza di también in una determinata Unità Informativa e l'attivazione di uno fra i suoi possibili valori d'impiego (funzione: avverbio focalizzante o connettivo testuale ${ }^{2}$; valore semantico: additivo, inclusivo o di uguaglianza). Partendo da alcuni studi sulla traduzione delle particelle attraverso il MB (Borreguero 2014 e De Cesare / Borreguero 2014), osserverò quali traducenti dell'italiano, fra anche, pure, inoltre ecc., siano stati selezionati dalla traduttrice, in modo da rinve-

\footnotetext{
Ricorro alla locuzione "particelle discorsive" come iperonimo che denota la macro-categoria nella quale sono inclusi gli elementi della lingua dotati di un significato procedurale e di una portata testuale. Le particelle discorsive, a loro volta, a seconda della funzione svolta (focalizzante o connettiva), del grado di integrazione (maggiore o minore) entro la struttura sintattica frasale e della natura (locale o testuale) della loro portata, sono denominate rispettivamente "focalizzatori (o avverbi focalizzanti)" e "connettivi testuali". Per "connettivi testuali" intendo gli elementi del lessico funzionale delle lingue dotati di una portata testuale che veicolano una connessione (additiva, concessiva, contrastiva ecc.) tra (parti di) enunciati incidendo sull'organizzazione logica e tematica del testo. Gli "avverbi focalizzanti", invece, maggiormente integrati nella struttura sintattica della frase, sono operatori transcategoriali e dotati di una semantica relazionale, nel nostro caso, additiva; costituiscono una variante terminologica degli "avverbi paradigmatizzanti” (cfr. Nølke 1983; De Cesare 2004: 215; Ferrari et al. 2008: 340). Pur riconoscendo che la caratteristica intrinseca di questi avverbi sia l'evocazione di un paradigma di alternative (Krifka 2007: 26; De Cesare 2010: 101; Andorno / De Cesare 2017: 159) e non la delimitazione del Fuoco Informativo di enunciato, la scelta di ricorrere al termine "focalizzante" ha una duplice ragione: da una parte, costituisce un'etichetta più trasparente in un'ottica interlinguistica (spagnolo: adverbios de foco e focalizadores; inglese: focus adverbs e focus markers), dall'altra, la possibilità di alternare le varianti sinonimiche "avverbi focalizzanti" e "focalizzatori" conferisce al testo una maggiore snellezza in termini stilistici.
} 
nire eventuali regolarità traduttive sulla base della segmentazione del MB. L'analisi proposta sarà fondamentalmente qualitativa; pertanto, $\mathrm{i}$ dati quantitativi forniti si limiteranno al resoconto delle occorrenze totali di también nel testo e dei principali traducenti riscontrati nella traduzione in italiano (cfr. nota 11).

Le particelle discorsive - il cui studio, a partire dalla fine del secolo scorso, ha prodotto moltissima bibliografia (per la lingua spagnola, si vedano, ad esempio: Fuentes 1987; Martín Zorraquino / Montolío 1998; Briz 1998; Carranza 1998; Pons Bordería 1998; Portolés 1998; Martín Zorraquino / Portolés 1999; Loureda / Acín 2010) - non sono più considerate alla stregua di meri riempitivi o supporti conversazionali. Infatti, se un tempo ci si riferiva a questi elementi della lingua tramite le etichette di bordones 'bastoni' (Valdés 1736: 135; Covarrubias 1611: 345) e di muletillas 'stampelle' (Gili Gaya 1943: 326), attualmente, le particelle discorsive sono considerate una categoria funzionale che ha un ruolo ben preciso entro l'economia testuale, ossia quello di guidare l'elaborazione delle inferenze durante la comunicazione (Martín Zorraquino / Portolés 1999: 4058). Si tratta, in sostanza, di parole (o locuzioni) dotate di un significato procedurale, non referenziale, che regolano il contesto pertinente per l'elaborazione delle inferenze a partire dall'informazione codificata negli enunciati su cui operano.

A riprova della loro importanza nell'architettura testuale, è stato dimostrato (Loureda / Cruz 2013; Nadal et al. 2016), mediante il monitoraggio dei movimenti oculari (eyetracking), che le particelle discorsive presentano costi di lettura - quindi di elaborazione (processing) - maggiori rispetto al lessico che veicola un significato proposizionale. In particolare, relativamente alla lingua spagnola, l'incremento dello sforzo cognitivo risulta considerevole nel caso del connettivo controargomentativo sin embargo e del focalizzatore additivo-scalare ${ }^{3}$ incluso, mentre non vi sarebbe un aumento significativo nel caso del connettivo additivo además (Nadal et al. 2016: 57).

Tale differenza, probabilmente, è da ascrivere alla diversa natura logica della connessione veicolata: la relazione additiva, infatti, s'iscrive nella naturale successione lineare di una proposizione e può, per questo, essere ridondante esplicitarla mediante un operatore. Dunque, il discrimine per valutare i diversi costi di elaborazione degli enunciati è dato non solo dal significato procedurale (vs. proposizionale) delle particelle discorsive, ma anche dalla natura, o meglio dalla complessità, della connessione veicolata. Per questa ragione, rispetto a uno stesso enunciato - ad esempio, Alicia sabe dividir -, i costi di elaborazione risultano significativamente maggiori con l'inserzione del focalizzatore additivo-scalare incluso (Alicia sabe incluso dividir) rispetto a quella del focalizzatore additivo también (Alicia sabe también dividir) che marca un'informazione convenzionale minore (Loureda / Cruz 2013: 84). Infatti, también può essere considerato «un "hiperónimo procedimental" de incluso» (Loureda / Cruz 2013: 93) nella misura in cui proietta una scala additiva ma

Per valore scalare intendo, seguendo Andorno (2000), Portolés (2007, 2010) e Andorno / De Cesare (2017: 163), l'ordinamento degli elementi di un paradigma all'interno di una scala di aspettative. È possibile distinguere almeno tre tipi di scale informative: (i) le scale semantiche, (ii) le scale pragmatiche e (iii) le scale proiettate dal significato convenzionale di alcuni lessemi. L'ordine interno alle scale semantiche dipende dai paradigmi lessicali e grammaticali di ciascuna lingua, in base ai quali l'elemento più forte comprende l'elemento più debole (Portolés 2007: 137). In secondo luogo, le scale pragmatiche dipendono dalla conoscenza del mondo e dagli specifici valori culturali di riferimento. Infine, l'ordinamento delle scale proiettate dal significato procedurale di alcuni lessemi avviene su base pragmatica, mentre la direzione delle inferenze (ammesse vs. non consentite) è guidata dal valore semantico del focalizzatore (Andorno 2000: 59). 
non culminativa, nella quale l'informatività corrisponde alla somma degli elementi che compongono il paradigma, a differenza di incluso che, invece, proietta una scala additiva e culminativa in base alla quale l'elemento annesso al paradigma è caratterizzato come il più informativo.

D'altra parte, l'esperimento di eyetracking dimostra pure che i tempi di lettura degli enunciati in cui compaiono le particelle discorsive sin embargo e incluso aumentano ulteriormente in seguito alla rimozione delle stesse. Infatti, eliminando le particelle - le quali marcano convenzionalmente il tipo di relazione fra (parti di) enunciati, regolando così l'accesso alla rappresentazione mentale pertinente - l'enunciato risulta in tal modo sotto-determinato e, per questo, l'elaborazione del suo significato presenta tempi di lettura maggiori. Resta tuttavia da verificare se l'analisi dei movimenti oculari nella traduzione darebbe costanti simili alla versione originale, e se la nostra categorizzazione basata sul MB è in grado di predire lo sforzo cognitivo, dunque, i tempi di lettura.

Il lessico funzionale della lingua costituisce, in sostanza, 1'area di maggiore attenzione e sforzo cognitivo durante l'interpretazione di un enunciato, poiché in esso sono iscritti i valori informativi e le relazioni logiche ${ }^{4}$ fondamentali per la sua comprensione. Queste considerazioni, sommate all'ormai attestata polifunzionalità paradigmatica di molte particelle discorsive, rendono la traduzione interlinguistica di queste parole tanto problematica quanto cruciale.

\section{Le particelle additive in spagnolo e in italiano}

La letteratura sulle particelle discorsive in lingua spagnola offre, allo stato attuale, una quantità di studi maggiore di quella disponibile per la lingua italiana, sia in virtù dello spazio concesso dalle grammatiche recenti dello spagnolo (GDLE 1999; NGLE 2009) ai fenomeni pragmatici sia in virtù della presenza di ben quattro dizionari specialistici dedicati all'analisi lessicografica delle particelle discorsive della lingua spagnola (Santos Río 2003; Briz / Pons / Portolés 2008; Fuentes 2009; Holgado 2017). Proprio in relazione alle voci registrate in questi dizionari, occorre segnalare l'assenza, nel DPDE (Briz / Pons / Portolés 2008), di una voce relativa a también. Tuttavia, in italiano si registrano diversi studi dedicati all'analisi di un sottoinsieme delle particelle discorsive: gli avverbi focalizzanti (p. es., Andorno 2000; De Cesare 2015; Andorno / De Cesare 2017; De Cesare 2019).

Uno dei valori semantici veicolati dal significato procedurale delle particelle discorsive - e dalla comunicazione umana in generale - è l'addizione, vale a dire, l'espressione di una relazione in cui un costituente è annesso, in modo esplicito o implicito, ad (almeno) un altro. Da una prospettiva informativa, la connessione additiva mette in relazione due (o più) elementi che sono Commenti a uno stesso Topic (Portolés 2010: 291; Sainz 2012: 50). Tuttavia, da una prospettiva di organizzazione del discorso, tale connessione può risultare un'operazione ridondante, dunque innecessaria, che non ha bisogno di un'esplicita codifica verbale ma che può essere realizzata mediante la semplice giustapposizione delle unità che si sommano

\footnotetext{
Ricorro alle espressioni "valori semantici" e "relazioni logiche" - quest'ultima, seguendo Ferrari et al. (2008: 149) - per indicare le relazioni di concessione, contrasto, addizione ecc. che sussistono fra (parti di) enunciati, le quali possono essere veicolate esplicitamente mediante il ricorso a determinate particelle discorsive.
} 
(Cuartero Sánchez 2002: 135; De Cesare / Borreguero 2014: 89). Ciononostante, le varie lingue naturali hanno sviluppato un'ampia gamma di espressioni specializzate nel marcare diversi tipi di operazioni additive che, ad esempio, possono implicare connotazioni epistemiche, o ancora, valutazioni gerarchiche, ordinando gli elementi su scale informative. Inoltre, sul piano del contenuto è pertinente attuare una distinzione fra due operazioni solo in apparenza simili, ma che in realtà presentano differenze sostanziali: da una parte, il valore additivo e, dall'altra, quello inclusivo o «de concreción» (Sainz 2012: 56). Se è vero che in entrambi i casi c'è un paradigma che ingloba una serie di unità, tuttavia, nel caso del valore inclusivo, un elemento funziona come termine incluso e un altro come termine inclusivo, mentre nel caso del valore additivo, un elemento è annesso a un altro per formare un paradigma di elementi posti sullo stesso piano.

Lo spagnolo también e l'italiano anche sono, nelle rispettive lingue, i focalizzatori più frequenti per l'espressione linguistica del valore additivo e inclusivo. Complessivamente, l'azione degli avverbi focalizzanti sull'organizzazione informativa dell'enunciato consiste nel focalizzare un costituente della frase evocando, al contempo, un'alternativa, sia essa esplicita o implicita, ammessa o esclusa (Krifka 2007: 30; NGLE 2009: 2985; Portolés 2010: 295; Borreguero 2011: 444; Sainz 2012: 41; De Cesare 2015: 33). Gli enunciati che contengono un avverbio focalizzante innescano così vari movimenti inferenziali: nello specifico, «implicano il contenuto della Proposizione senza l'avverbio e presuppongono una Proposizione che contiene una variabile aperta al posto del segmento modificato dall'avverbio» (Ferrari et al. 2008: 342). Se invece i focalizzatori vengono eliminati, l'inferenza della proposizione alternativa, qualora essa non sia codificata linguisticamente, scompare (Sainz 2012: 52). In effetti, la creazione di un paradigma di alternative è talmente centrale che la classificazione dei diversi tipi di focalizzatori si basa proprio sulla relazione stabilita tra il fuoco dell'avverbio e il paradigma di alternative possibili (Andorno 2000: 49; GRAE 2009: 2992). Così, gli avverbi additivi (p. es., sp. también e it. anche) determinano la validità delle alternative, mentre quelli restrittivi (p. es., sp. solo e it. soltanto) escludono le alternative.

I focalizzatori, inoltre, possono combinare la funzione di avverbio focalizzante con quella di connettivo testuale (De Cesare 2019a: 92). Si tratta di una tendenza interlinguistica dei focalizzatori - non limitata a quelli con valore additivo - a operare come connettivi, estendendo la propria portata da un sintagma della frase alla connessione tra enunciati (Andorno 2000: 99). La prossimità tra focalizzatori e connettivi e, più in generale, la relazione tra connessione e focalizzazione è stata segnalata, fra gli altri, da Portolés (2010: 298) e da Sainz (2012: 42). In particolare, Portolés (2010: 298) osserva che lo studio degli avverbi focalizzanti è all'origine di buona parte delle considerazioni attuali sul significato informativo delle particelle discorsive (marcadores del discurso), dal momento che il loro comportamento testuale presenta numerose analogie ${ }^{5}$.

L'obiettivo principale di un'analisi contrastiva delle particelle discorsive consiste nell'identificazione delle caratteristiche contestuali e cotestuali che, in certi casi, fa-

\footnotetext{
Per questa ragione, nel Diccionario de partículas discursivas del español (DPDE) (Briz / Pons / Portolés 2008) - dizionario online e in fieri: <http://www.dpde.es> - sia «los focalizadores» che «los marcadores del discurso» sono considerati «partículas discursivas», dove per «partícula» è intesa: «cualquier palabra invariable o locución que guíe por su significado el procesamiento de otra unidad con significado conceptual» (Portolés 2010: 298).
} 
voriscono un'equivalenza e, in altri, la impediscono. Ad esempio, le analogie tra lo spagnolo también e l'italiano anche si basano sulla comune funzione di avverbi focalizzanti dotati di un valore semantico additivo e inclusivo. Tuttavia, come si vedrà (cfr. $§ 2.1$ ), también e anche differiscono: (i) per il tipo di istruzione semantica additiva, che deriva dal diverso processo di grammaticalizzazione, (ii) per l'attivazione del valore scalare, (iii) per la compatibilità con la funzione di connettivo testuale e (iv) per la posizione sintattica ammessa all'interno della frase, più precisamente, rispetto al fuoco dell'avverbio. I criteri sintattici, tuttavia, non bastano per spiegare la loro incidenza sul piano sintagmatico (Borreguero 2014: 15). Al contrario, 1'individuazione di particolari posizioni enunciative (o «structural slots» per Fischer 2006: 430) e delle corrispondenti funzioni discorsive, come proposto dal Modello di Basilea (cfr. § 3), sembra consentire un'approssimazione più accurata agli usi co(n)testuali delle diverse particelle discorsive, anche in un'ottica interlinguistica.

\subsection{También vs anche}

L'espressione della relazione additiva può comportare un parallelismo informativo, semantico e sintattico fra gli elementi del paradigma (Sainz 2006: 44). Dal punto di vista del valore semantico, tale parallelismo si traduce sovente in un'istruzione semantica di uguaglianza, la quale è iscritta nel significato procedurale di también, ma non in quello di anche. La causa di tale differenza va cercata nella diversa base lessematica dei focalizzatori delle due lingue (Sainz 2006: 29). All'origine di también vi è infatti la struttura comparativa "tan bien como", la quale si è poi grammaticalizzata come avverbio che riconosce l'uguaglianza fra i termini comparati (Sainz 2006: 30). Diversamente, nel suo significato pre-grammaticalizzazione, anche equivaleva ad 'ancora', 'finora', 'del resto', ecc. (De Cesare 2004a: 4); in effetti, da un punto di vista etimologico, anche e anco sono delle semplici varianti di ancora (Ricca 2017: 70). Nell'uso contemporaneo di anche permangono tracce del significato originario che si manifestano nella convivenza del valore additivo con quello concessivo (De Cesare 2004a: 23), di cui è prova, ad esempio, l'uso della locuzione concessiva anche se (De Cesare 2004a: 26), uso decisamente attuale e non arcaico. Di fatto, nell'italiano antico (XIII-XIV sec.), le locuzioni concessive più frequenti erano se pure ed eziandio se, mentre anche se aveva un valore unicamente additivo, equivalente all'odierno inoltre (Ricca 2017: 63). A causa del diverso processo di grammaticalizzazione, dunque, la connessione additiva realizzata da también e da anche veicola delle istruzioni semantiche sostanzialmente diverse.

Così, mentre il focalizzatore sp. también attua un'operazione additiva che comporta l'uguaglianza (sintattica e informativa) fra i termini del paradigma, l'it. anche veicola un significato additivo più vicino alla concessione argomentativa. A causa della diversa natura dell'istruzione additiva, también e anche sono intercambiabili soltanto quando si rispetta l'istruzione comparativa di uguaglianza, quindi di coordinazione sintattica (Sainz 2006: 33). Questa restrizione, ad esempio, è alla base dell'inaccettabilità della sequenza *también porque, mentre la stessa (anche perché) è perfettamente accettabile in italiano: la focalizzazione di un complemento subordinato, infatti, violerebbe l'istruzione comparativa di uguaglianza iscritta nel significato di también (Sainz 2006: 34).

Per quanto riguarda la distribuzione sintattica, anche compare di preferenza prima del sintagma nominale e dopo il sintagma verbale, oppure tra l'ausiliare e il par- 
ticipio, ma mai prima del verbo (De Cesare / Borreguero 2014: 61), a meno che non si tratti di una forma verbale di modo indefinito (participio, infinito, gerundio). In particolare, quando il fuoco di anche è un soggetto sintattico, l'avverbio è collocato di preferenza come pre-modificatore lineare, ossia immediatamente a sinistra del soggetto (De Cesare 2015: 36). Diversamente, lo sp. también è dotato di una grande mobilità posizionale, vale a dire, può operare sia come pre-modificatore sia come post-modificatore, indipendentemente dalla categoria grammaticale del suo operando ${ }^{6}$ (Santos Río 2003: 616). Tuttavia, secondo la Real Academia Española (NGLE 2009: 2992), in presenza di un gruppo verbale, la posizione canonica di también è quella di pre-modificatore. Inoltre, il fuoco di también può essere contiguo oppure a distanza (Kovacci 1999: 774). Questa libertà posizionale produce sovente ambiguità circa la determinazione del dominio di associazione dell'avverbio; ambiguità che, come si vedrà (cfr. $\S 4.2$ ), è perlopiù superficiale e di natura sintattica, e può essere risolta ricostruendo la struttura comparativa soggiacente, ossia lo schema proiettato dal focalizzatore e che consiste nel (i) termine presupposto che fa da sfondo comune al (ii) paradigma di alternative, codificate o implicite, a cui si annette (iii) il fuoco dell'avverbio.

Dunque, dal punto di vista sintattico, le differenze principali tra también e anche riguardano innanzitutto la possibile collocazione a distanza rispetto al loro fuoco, la quale è possibile per también ma non per anche, che è invece posto quasi esclusivamente a ridosso del suo fuoco, obbedendo così al principio del fuoco ristretto (narrow scope position) (De Cesare 2015: 36). Secondariamente, i focalizzatori delle due lingue divergono per la posizione ammessa - anteposta o posposta - rispetto al loro operando. Anche in questo caso, también gode di maggiore libertà posizionale rispetto ad anche, la cui distribuzione è vincolata alla categoria grammaticale del suo operando. In particolare, il principio del fuoco a destra (the principle of right scope) rispetto a un elemento nominale ( $v s$ verbale) è una caratteristica attestata del comportamento distribuzionale di anche, che tende a operare come post-modificatore di un nome soltanto in configurazioni marcate e con enfasi intonativa su anche (De Cesare 2015: 37).

Il principio del fuoco a destra si manifesta pure nella frequente ripresa pronominale dopo l'avverbio del referente su cui anche opera (De Cesare 2015: 40). Peraltro, da un punto di vista informativo, la ripresa pronominale dopo l'avverbio risolve il conflitto originato da un soggetto preverbale che funzioni, al contempo, come Topic e come Focus di frase (De Cesare 2015: 41). Questo conflitto viene risolto distribuendo le due funzioni informative entro due sintagmi nominali diversi; più precisamente, il Topic ricade sul soggetto preverbale, mentre il Focus è associato al pronome postverbale preceduto da anche, come nell'esempio proposto da De Cesare (2015: 41): «Stella ${ }_{\text {Topic }}$ legge anche le $i_{\text {Focus }}{ }^{7}$. Tuttavia, se dopo il verbo inseriamo un complemento, sia esso diretto o indiretto, la ripresa pronominale dopo l'avverbio

Ricorro alle etichette "fuoco (dell'avverbio)", "operando", "portata", "dominio di associazione" e "dominio operativo" e per riferirmi alla porzione testuale su cui la particella proietta la propria semantica relazionale e con la quale si combina sintatticamente o, in altre parole: «the element that is to be interpreted as added» (De Cesare 2015: 34).

Simboli e notazioni convenzionali: testo ${ }_{\text {fuoco }} \rightarrow$ fuoco dell'avverbio; testo ${ }_{\text {Focus }} \rightarrow$ Fuoco di enunciato; testo Topic $_{\text {. }}$ $\rightarrow$ Topic di enunciato; testo ${ }_{\text {Comment }} \rightarrow$ Comment di enunciato; $\mathrm{MB} \rightarrow$ Modello di Basilea; UC $\rightarrow$ Unità Comunicativa; UI $\rightarrow$ Unità Informativa; /Quadro /Nucleo/Appendice $\rightarrow$ Confini di UI; // $\rightarrow$ Confine di enunciato; [testo] ${ }^{\text {Inciso }} \rightarrow$ Inciso; grassetto $\rightarrow$ segmento evidenziato a fini descrittivi. 
non è più una mera strategia per distribuire l'informazione in blocchi separati; al contrario, diventa necessaria per non spostare sul complemento il fuoco di anche: Stella ${ }_{\text {Topic }}$ legge anche le $i_{\text {fuoco }}$ Shakespeare ${ }_{\text {Focus }}$. In effetti, in base al principio del fuoco a destra, in assenza di ripresa pronominale: Stella ${ }_{\text {Topic }}$ legge anche Shakespeare ${ }_{\text {fuoco, }}$, anche proietterebbe il suo dominio di associazione sul complemento "Shakespeare", evocando così un diverso insieme di elementi in gioco, dove l'alternativa al fuoco dell'avverbio sarebbe data non dalle "persone che leggono Shakespeare" bensì dagli "autori letti da Stella".

La configurazione locale del focalizzatore anche è alla base di un'ulteriore differenza con lo sp. también, vale a dire, la possibilità di operare come connettivo dotato di una portata testuale, tipicamente, nell'occorrenza in periferia sinistra di enunciato. Mentre también si colloca con frequenza alla periferia, sia sinistra che destra, dell'enunciato (Borreguero 2011: 448), anche non può occupare la posizione iniziale preverbale $^{8}$ e quindi avere l'intero enunciato nella sua portata (Andorno 2000: 100). Inoltre, a causa del principio del fuoco ristretto, quando anche occupa la posizione iniziale pre-nominale, il suo ambito operativo tende a limitarsi al fuoco dell'avverbio, senza proiettarsi sul resto dell'enunciato. In virtù di tali restrizioni sintattiche, l'uso di anche in funzione di connettivo testuale è considerato molto marginale (De Cesare 2004: 195) oppure del tutto assente (Andorno 2000: 100; Borreguero 2011: 456). Per espletare la funzione connettiva, la lingua italiana si avvale perciò di altri elementi come inoltre, in più, in aggiunta ecc. (Borreguero 2011: 456). D'altronde, in presenza di competitori linguistici, i lessemi semanticamente neutri sono più frequenti di quelli marcati (Andorno 2000: 85), ragion per cui la connessione additiva tra enunciati è realizzata più sovente dai sopraccitati inoltre ecc., che sono connettivi intrinsecamente testuali.

Invece, la maggiore libertà posizionale di también e, in particolare, la possibilità di risalire nella periferia sinistra, di precedere il verbo e di operare su un fuoco a distanza fanno sì che l'avverbio spagnolo possa operare come connettivo testuale. Malgrado alcune riserve in sede teorica (Cuartero Sánchez 2002: 175; Santos Río 2003: 616), infatti, la maggior parte degli studi (p.es., Kovacci 1999: 770; Fuentes 2009: 331; Borreguero 2011: 453) ammette l'uso di también come connettivo testuale.

Infine, relativamente all'attivazione del valore scalare, che non costituisce una caratteristica intrinseca né di anche né di también - a differenza, per esempio, dell'it. perfino (Visconti 2005: 239) e dello sp. incluso (Portolés 2010: 309) -, anche risulta compatibile con un'interpretazione scalare (De Cesare 2019: 165) più di quanto lo sia también (Borreguero 2011: 445; Calvi 2016: 149). Se da una parte Cuartero Sánchez (2002: 313) e Sainz (2006: 30) escludono l'attivazione di una scala di valori in base alla quale también introdurrebbe l'informazione più saliente della serie, invece, altri autori (Santos Río 2003: 616; Borreguero 2011: 445; Calvi 2016: 149) ritengono che, sebbene non costituisca un tratto inerente all'avverbio, fattori contestuali e intonativi possono attivare un'interpretazione scalare. D'altronde, i valori scalari, ove non inerenti alla semantica dell'avverbio, possono essere contestualmente determinati (Andorno 2000: 56), tuttavia, devono comunque essere compatibili con le

Come si è detto, fanno eccezione le forme verbali di modo indefinito (participio, infinito e gerundio); tuttavia, in caso di anteposizione rispetto a un gerundio, il valore associato ad anche è prevalentemente concessivo, non additivo: anche volendo, non posso. 
istruzioni semantiche iscritte nel significato procedurale dell'avverbio. Per questa ragione, ossia a causa dell'istruzione comparativa di uguaglianza - inerente alla semantica di también ma non a quella di anche (Sainz 2006: 30) -, l'eventuale attivazione del valore scalare da parte di también è fortemente ancorata al contesto e, in ogni caso, la scala di valori proiettata non può essere una scala culminativa. In altre parole, también attiva una lettura di equivalenza o di uguaglianza (A come B) che deriva, molto probabilmente, dalla base lessicale: il sintagma comparativo "tan bien como"; pertanto, solo fattori contestuali possono innescare una lettura di A come meno probabile, dunque più informativo, di B.

\section{Il Modello di Basilea per la segmentazione pragmatica del testo}

La categoria funzionale delle particelle discorsive appartiene al dominio del discorso e non a quello della frase: la loro funzione connettiva, infatti, non opera sui legami sintattici bensì sui processi inferenziali. Dall'appartenenza al dominio discorsivo deriva l'impossibilità, o quanto meno la scarsa utilità, di un'analisi formale in base alle categorie della sintassi tradizionale, nonché la conseguente necessità di individuare delle unità per la segmentazione del discorso. Infatti, se da un lato c'è una sintassi arbitraria governata da principi formali come l'accordo tra il soggetto e il predicato verbale, dall'altro ce n'è una motivata da ragioni pragmatiche (Ferrari et al. 2008: 30; Borzi 2009: 81). Per identificare i costituenti di una sintassi motivata da ragioni pragmatiche occorre mettere da parte - sia pur parzialmente e da un punto di vista perlopiù quantitativo - il criterio grammaticale in favore di quello pragmatico, affinché la sintassi frasale lasci il campo alla sintassi discorsiva, da cui emergerebbero nuove categorie discrete attraverso le quali osservare la correlazione fra le particelle discorsive e la loro posizione all'interno del discorso, la cui segmentazione è un'operazione fondamentalmente inferenziale (Ferrari 2004: 10).

In questo senso, il modello di segmentazione testuale messo a punto dalla scuola basilese (Ferrari et al. 2008) costituisce, al momento, l'unico modello per la segmentazione pragmatica del testo scritto ${ }^{9}$. Sebbene la lingua di applicazione del MB sia l'italiano, tuttavia, Borreguero (2014) e De Cesare / Borreguero (2014) ne hanno dimostrato la validità anche per l'analisi di alcune particelle discorsive dello spagnolo e del francese.

Dal punto di vista teorico, il MB considera che nella componente semantica del lessico, della sintassi e della punteggiatura siano codificati valori testuali e che, pertanto, le scelte microlinguistiche siano definite e controllate dalla testualità. L'attualizzazione comunicativa dei valori testuali iscritti nella lingua sarebbe mediata dall'articolazione informativa della frase, la quale svolgerebbe un ruolo di interfaccia tra due sistemi di organizzazione della comunicazione verbale: quello linguistico e quello testuale. I valori testuali codificati nella lingua sarebbero quindi «astratti e sotto-specificati», nel senso che se ne può misurare l'impatto interpretativo solo

La segmentazione del parlato, invece, può fare affidamento su diversi modelli. Ad esempio, fra quelli elaborati per le lingue romanze, ci sono: il "Geneva Model" (Roulet et al. 2001); il "Fribourg Model" (Berrendonner 2012); il "Val.Es.Co Model" (Briz et al. 2003); il "Co-enunciation Model" (Morel 2010); il "Prominence Demarcation Model" (Lombardi Vallauri / Tamburini 2012); e il "Basic Discourse Units Model" (Degand / Simon 2009). Alcuni degli studi realizzati mediante i diversi modelli di segmentazione del discorso disponibili per le lingue romanze, compreso il MB, sono raccolti in Pons Bordería (2014). 
nel momento in cui si realizzano comunicativamente, vale a dire, in un determinato contesto d'uso (Ferrari et al. 2008: 26). Un ruolo centrale nell'organizzazione del modello, dunque, è svolto dalla struttura informativa della frase, considerata come «quella zona della costruzione del significato comunicativo che è (in buona parte) controllata dalla lingua e che, nel contempo, controlla (in buona parte) l'organizzazione semantico-pragmatica del testo» (Ferrari et al. 2008: 21).

Nel MB la porzione testuale principale è costituita dal Capoverso, che costituisce l'Unità Comunicativa caratterizzata da una funzione illocutiva e da una funzione testuale, e la cui articolazione linguistica consiste in una sequenza di Enunciati, o al limite anche di uno solo, delimitati da uno spazio (Ferrari et al. 2008: 33); il Capoverso intrattiene con gli altri Capoversi una o più relazioni referenziali e tematiche, logico-argomentative, enunciativo-polifoniche e tipologiche (Ferrari et al. 2018: 25). L'Unità Comunicativa inferiore al Capoverso è costituita dal Movimento Testuale, inteso come un insieme di Enunciati dotati di qualche tipo di unitarietà logica o composizionale all'interno dell'architettura testuale. In terzo luogo, c'è l'Enunciato, termine con cui si designa, da una prospettiva comunicativa, '’entità grammaticale costituita dalla frase. Le Unità Comunicative che formano il Capoverso si distribuiscono su uno spazio semantico-pragmatico tridimensionale al cui interno «si riconoscono un piano principale e un (uno o più) piano secondario, collocato in profondità rispetto al piano principale» (Ferrari et al. 2008: 34). Fra questi, solo il primo piano è fondamentale e necessario, mentre i piani secondari, costituiti dalle Unità Comunicative caratterizzate come Incisi - che marcano una discontinuità, rispetto al primo piano, nelle dimensioni: enunciativa, logica e/o referenziale - sono facoltativi.

Secondo questo modello di architettura testuale, i segni di punteggiatura posti al confine tra le Unità Comunicative rappresentano «il segno della proiezione linguistica bidimensionale di un'organizzazione la cui natura profonda è tridimensionale» (Ferrari et al. 2008: 35-36). L'assetto interpuntivo, nello specifico, è inteso in chiave eminentemente comunicativo-testuale; pertanto, le eventuali regolarità sintattiche e prosodiche sono considerate epifenomeni di una realtà fondamentalmente comunicativa (Ferrari et al. 2018: 16). Infatti, la punteggiatura italiana contemporanea, al pari di quella spagnola (Lala 2015: 69), risponde a una ratio comunicativa (vs morfosintattica o pausativa). Per questa ragione, i segni interpuntivi nelle due lingue qui prese in considerazione, oltre a marcare confini formali, consentono di segmentare il testo nelle sue unità semantico-pragmatiche costitutive, di gerarchizzare l'informazione veicolata e di fornire indicazioni su come interpretare tali segmenti sia in prospettiva testuale sia in prospettiva interattiva (Ferrari et al. 2018: 19).

L'Unità Comunicativa minima fondamentale del $\mathrm{MB}$, dunque, è rappresentata dall'Enunciato, la cui organizzazione informativa è definita da un insieme di proprietà rappresentabili da altrettanti livelli di articolazione informativa. Questi livelli informativi sono: (i) il livello dell'articolazione Topic-Comment, costruito attorno alla relazione di aboutness referenziale - ossia di genitoralità semantica - vigente tra un referente testuale e la Proposizione Semantica in cui compare; (ii) il livello dell'articolazione gerarchico-informativa, la cui funzionalità è prettamente testuale; (iii) il livello di attivazione cognitiva dei referenti testuali nella Memoria Testuale, definito dalle proprietà "Attivo", "Semiattivo" e "Inattivo".

L'articolazione del livello gerarchico-informativo all'interno dell'Enunciato può essere intesa come «un'architettura di "spazi" gerarchicamente ordinati e saturati 
da materiale semantico-pragmatico, che coincidono con le unità minimali del testo» (Ferrari et al. 2008: 88). Queste unità minimali del testo corrispondono alle Unità Informative di Nucleo, Quadro e Appendice ${ }^{10}$, la cui gerarchia è stabilita in base alla loro specializzazione informativo-testuale. Le frontiere tra un'Unità Informativa e l'altra possono essere individuate sulla base di specificità sintattiche, lessicali e di uso della punteggiatura. Non sono presi in considerazione, invece, fattori legati all'andamento prosodico, dal momento che si tratta di un modello di segmentazione testuale elaborato per l'analisi della lingua scritta.

Delle tre Unità Informative, il Nucleo è l'unica sufficiente e necessaria affinché l'Enunciato sussista. Il Nucleo contiene le relazioni logiche e topicali principali, inoltre, definisce la funzione illocutiva dell'Enunciato e ospita il Focus informativo, ossia la parte comunicativamente più rilevante, che, nella sua manifestazione non marcata, in base al principio dell'end-Focus, si colloca a destra nel Nucleo (De Cesare 2010: 108). Rispetto all'articolazione Topic-Comment, il Nucleo può coincidere con una proposizione articolata in Topic-Comment, come in (1), ma può anche contenere il solo Comment, laddove il Topic sia implicito oppure realizzato in posizione arretrata nel Quadro, come in (2), dove l'emarginazione a sinistra del Topic serve a rendere più trasparente il valore focale del Comment, a cui viene sottratto il materiale residuale:

(1) // / Le cause più comuni di ostruzione delle vie alte respiratorie ${ }_{\text {Topic }}$ sono rappresentate dalla ipertrofia del tessuto linfatico e dalle riniti croniche ${ }_{\text {Comment }}{ }^{/ N u c l e o} / / \mathrm{E}$ (2) // / Dei malviventi ${ }_{\text {Topic }} / /$ Quadro invece, $/ /^{\text {Appendice non }} \mathbf{c}^{\prime}$ è traccia ${ }_{\text {Comment }} /{ }^{\text {Nucleo }} / /_{\mathrm{E}}$ (ess. tratti da Ferrari et al. 2008: 92)

A differenza del Nucleo, il Quadro è facoltativo e la sua posizione, rigida: quando viene realizzato, precede il Nucleo aprendo l'Enunciato. Il particolare contributo che il Quadro offre alla costruzione dell' architettura testuale consiste nell' esplicitazione del contenuto sullo sfondo del quale il Nucleo deve essere interpretato. Per quanto concerne il suo contenuto semantico, il Quadro tipicamente: (i) veicola indicazioni circostanziali; (ii) indica l'ambito cognitivo in cui si definisce la verità del referente nucleare; (iii) veicola l'atteggiamento epistemico o assiologico del locutore nei confronti del referente nucleare; (iv) esprime la fonte enunciativa del contenuto nucleare; (v) accoglie valori intrinsecamente testuali e non denotativi, come quelli associati ai connettivi. La pertinenza dei contenuti semantici del Quadro, dunque, è, in maniera diretta o indiretta, di natura fondamentalmente testuale e si manifesta sia nei confronti del cotesto di sinistra sia verso quello di destra. Per quanto riguarda il cotesto di sinistra, il Quadro assicura soprattutto «la coerenza e la consistenza referenziale della progressione semantico-pragmatica del testo» (Ferrari et al. 2008: 102-103). Relativamente al cotesto di destra, invece, l'unità di Quadro ha «un'importante funzione strutturante, legata al fatto che essa "apre" spazi semantico-pragmatici (denotativi, enunciativi, topicali, logici ecc.) che restano validi per gli Enunciati successivi fino a indicazione diretta del contrario» (Ferrari et al. 2008: 103).

10 In una versione precedente del modello (cfr. Ferrari 2004), le tre Unità Informative che compongono l'Enunciato sono: Rema, Tema e Appendice, che corrispondono, grosso modo, rispettivamente al Nucleo, al Quadro e all'Appendice della versione finale (cfr. Ferrari et al. 2008; Ferrari et al. 2018). 
Ad esempio, l'azione del Quadro sul cotesto di destra si manifesta tipicamente quando accoglie una fonte enunciativa o un dominio concettuale di ragionamento, come in (3), dove il Quadro fornisce la cornice all'interno della quale interpretare il contenuto nucleare. Inoltre, in caso di giustapposizione di unità riempite da materiale di carattere denotativo, si ha una coordinazione informativa di Quadri se i contenuti appartengono a tipologie diverse (spaziali, temporali, agentive ecc.), come in (4). Diversamente, se le indicazioni possono essere ricondotte alla medesima tipologia semantica si ottiene la configurazione informativa Quadro-Appendice, come in (5):

(3) // / Sotto il profilo dell'etnologia africanista, /Quadro [// - per tradizione collegata in Italia all'esperienza coloniale o all'attività missionaria $-/ /]^{\text {Inciso }} 1$ 'Università di Torino ha registrato senza alcun dubbio un ritardo rispetto agli altri atenei italiani. /Nucleo $/ /_{\mathrm{E}}$

(4) // / Intorno alle tre, /Quadro1 a forza di spallate, /Quadro2 i prigionieri hanno sfondato la porta. $/$ Nucleo $/ /_{\mathrm{E}}$

(5) // / A Trenzano /Quadro nella Bassa, /Appendice Cesare Righetti [...] ha deciso di provare la moto di un amico. / Nucleo // (ess. tratti da Ferrari et al. 2008: 99-101)

Infine, a differenza del Nucleo e analogamente al Quadro, 1'Unità Informativa di Appendice è facoltativa e subordinata. È inoltre caratterizzata da una grande mobilità all'interno dell'Enunciato: può, infatti, seguire l'unità da cui dipende (un Quadro, un Nucleo o un'altra Appendice) oppure manifestarsi al suo interno, creando un fenomeno di discontinuità informativa (Ferrari et al. 2008: 105). La differenza fondamentale tra le due posizioni ammesse consiste nel diverso dinamismo comunicativo: minore se l'Appendice crea un fenomeno di discontinuità informativa, maggiore se segue linearmente l'unità da cui dipende. Per questo motivo, se si vuole sviluppare testualmente un'Appendice, la posizione naturale è quella conclusiva. Oltre alla diversa distribuzione sintagmatica, la differenza principale tra il Quadro e l'Appendice consiste non tanto nel materiale semantico quanto nella diversa portata dell'azione informativa: più o meno ampia per il Quadro, strettamente locale per l'Appendice (Ferrari et al. 2008: 106). Tuttavia, esiste una configurazione particolare in cui la portata dell'Appendice si può estendere all'intero Enunciato: quando segue il Quadro ed è saturata da materiale non denotativo, come quello associato agli avverbi di frase e ai connettivi. Fatta questa eccezione, da ascrivere in parte alle specificità dell'unità di Quadro a cui si lega, in parte al valore intrinsecamente testuale delle particelle discorsive, l'Appendice difficilmente proietta i suoi effetti testuali oltre i confini dell'unità a cui si lega. Per questa ragione, laddove la struttura globale dell'Enunciato lasci una scelta: «la presentazione di un'informazione come Appendice può essere dettata proprio dalla volontà di "spegnerne localmente" gli effetti semantico-pragmatici» (Ferrari et al. 2008: 107). Di conseguenza, l'Appendice è lo spazio ideale per arricchire il messaggio testuale senza complicare l'architettura degli enunciati, spostando così la complessità concettuale della costruzione del testo dalla connessione tra enunciati alla connessione intra-enunciativa (Ferrari et al. 2008: 109).

Ad esempio, l'Appendice può introdurre, come in (6), un ulteriore argomento senza, con ciò, intaccare l'argomentazione principale, proprio perché costituisce uno spazio convenzionalmente destinato a esaurire i suoi effetti comunicativi a livello locale. O ancora, può, come in (7), annullare localmente inferenze pragmatiche 
possibili ma non volute dal locutore, e offrire informazioni interpretativamente utili, forse note, ma che non si vuole dare per presupposte, come in (8):

(6) // / Da quando nel 2005 ho lasciato la mia cattedra di docente a Harvard e sono tornato in Canada, $/$ Quadro perché amo il mio paese, $/{ }^{\text {Appendice }}$ continuo a tornare sulla débâcle irachena. Nucleo //

(7) // / La storia della ricerca archeologica è fatta di tante sconfitte, /Nucleo che comunque non sono mai totali, $/ /^{\text {Appendicel }}$ perché apportano sempre nuove informazioni scientifiche. /Appendice $/ /{ }_{\mathrm{E}}$

(8) // / Bernardino Libonati, / Nucleo-il presidente di garanzia incaricato di traghettare la compagnia verso la vendita in occasione dell'asta al Tesoro, ${ }^{\text {Appendice }}$ ha dato ieri le dimissioni. -Nucleo $/ /_{\mathrm{E}}$ (ess. tratti da Ferrari et al. 2008: 106-108)

\section{Il MB per l'analisi contrastiva delle particelle additive}

Lo statuto informativo di una particella discorsiva, ossia la sua collocazione entro le unità di Nucleo, Quadro o Appendice, costituisce un fattore decisivo per la determinazione delle funzioni discorsive, dei valori d'impiego e della portata dei suoi operandi. Infatti, le proprietà informative si combinano con la semantica della particella innescando particolari effetti interpretativi. Come dimostrato dagli studi di Borreguero (2014) sullo sp. incluso e sui suoi traducenti in italiano e da quelli di De Cesare / Borreguero (2014) sullo sp. también, sul fr. aussi e sull'it. anche, alla collocazione di una particella discorsiva entro una specifica UI è riconducibile il variare di una serie di parametri che sono determinanti per definire l'impatto della particella sull'organizzazione semantico-logica del testo. Così, l'occorrenza entro ciascuna posizione enunciativa permette di orientarsi nella frequente polisemia e polifunzionalità delle particelle, facendo prevalere un significato (denotativo $v$ s procedurale), una funzione (avverbio focalizzante vs connettivo testuale) e un valore semantico (additivo, inclusivo, concessivo ecc.) particolari. Ad esempio, la collocazione di una particella nel Nucleo, specie se in posizione focale, è spesso il discrimine per l'attribuzione del valore denotativo rispetto a quello di connettivo testuale (Ferrari et al. 2008: 369). Ora, nel nostro specifico caso questa possibilità non sussiste: también, così come anche, è infatti caratterizzato da un certo grado di polifunzionalità ma non da polisemia in questo senso. Diverso è il caso, per esempio, dello sp. encima e dell'it. così, dove la collocazione nel Nucleo (vs Quadro) tende a far prevalere, rispettivamente, il valore di locuzione preposizionale di luogo (vs connettivo additivo-scalare) e quello di avverbio di modo (vs connettivo consecutivo).

Di seguito, attraverso l'impalcatura teorica del $\mathrm{MB}$, analizzerò le occorrenze di también e dei suoi traducenti in italiano ${ }^{11}$ estratti dal testo letterario ${ }^{12}$ El mal de

1 Le occorrenze totali di también nel testo sono 170. Nella versione in italiano si sono riscontrati i seguenti traducenti:136 anche; 14 pure; 4 a mia/sua volta; 3 pur; 3 sempre; 1 al contempo; 1 e poi; 1 nuovamente; 1 insieme. Per ultimo, si segnalano 4 occorrenze di también non tradotte, e 2 occorrenze tradotte non mediante una particella bensì convogliando la semantica relazionale dell'avverbio in un altro costituente della frase, ad esempio, nel verbo.

12 Come già accennato nell'Introduzione, si tratta di un testo dalla difficile collocazione entro un genere particolare. Infatti, se da una parte costituisce indubbiamente un testo di finzione narrativa catalogabile come romanzo, dall'altra presenta pure tratti tipici della tradizione diaristica e della saggistica di critica letteraria. Per questo, si 
Montano (Vila-Matas 2002) e dalla sua traduzione Il mal di Montano (Vila-Matas 2005). Le citazioni saranno riportate con le iniziali dell'autore (VM) seguite da (a) per il testo originale in spagnolo e (b) per il testo tradotto in italiano, e dal numero di pagina.

\subsection{También nelle UI}

La collocazione di una particella discorsiva nel Nucleo, come si è visto, tende (i) a favorire l'attivazione dell' eventuale significato denotativo ( $v s$ procedurale), (ii) a far prevalere, tra le due funzioni possibili associate al significato procedurale, quella di avverbio focalizzante ( $v s$. connettivo testuale) e (iii) a far coincidere, in molti casi, il fuoco dell'avverbio con il Fuoco Informativo della frase (De Cesare 2010: 108). Quando occorre nel Nucleo, dunque, la portata di también è limitata al fuoco dell'avverbio e può, pertanto, essere tradotto in italiano con anche (9) o con la sua variante pure (10). Come si evince dagli esempi, infatti, también non realizza una connessione tra enunciati, bensì opera, in (9) ${ }^{13}$, su un sintagma verbale ("es una metáfora perfecta") e, in (10), su uno nominale ("el nombre de Alina"):

(9a) // / Quizá la literatura sea eso: /Nucleo inventar otra vida que bien pudiera ser la nuestra, /Nucleo inventar un doble. /Nucleo // / Ricardo Piglia dice que recordar con una memoria extraña es una variante del doble, / Nucleo pero es también una metáfora perfecta de la experiencia literaria. / Nucleo // (VMa: 16) (9b) // / Forse la letteratura è questo: /Nucleo inventare un'altra vita che potrebbe benissimo essere la nostra, /Nucleo inventare un doppio. / Nucleo // ${ }_{E 1} / /$ / Ricardo Piglia dice che ricordare con una memoria estranea è una variante del doppio, /Nucleo ma è anche una metafora perfetta dell'esperienza letteraria. /Nucleo //

(VMb: 12)

può parlare di un testo ibrido (o trans-generico) che s'inserisce in una tradizione ormai attestata nella letteratura ispano-americana degli ultimi decenni, dove la cornice finzionale funge da pretesto per elaborare una critica letteraria non sistematica, vòlta a mostrare il dialogo costante dell'autore con la propria biblioteca personale (Esposito 2019: 251). Questo dialogo eterodosso con il canone trova il proprio asse centrale nel lascito letterario di Jorge Luis Borges. In sostanza, si tratta di una versione moderna di quello che già Alfonso Reyes (1944: 44) aveva identificato come «literatura ancilar», ossia un modo di fare critica attraverso «un prestito poetico totale» (Reyes 1962: 333) e senza compromessi metodici.

13 Relativamente all' $\mathrm{E}_{2}$ dell'esempio (9), la scelta di considerare le due unità che lo compongono due Nuclei, e non un Nucleo seguito da un'Appendice, poggia non tanto sulla struttura coordinante introdotta dalla congiunzione pero ( $m a$ in italiano), quanto sulla relazione di aboutness ivi espressa. Nel primo Nucleo, infatti, sono espressi il Topic ("recordar con una memoria extraña") e il primo Comment ("es una variante del doble"), mentre nel secondo Nucleo è espresso il secondo Comment ("es una metáfora perfecta de la experiencia literaria") dello stesso Topic. Da ciò deriva che i contenuti dell'Enunciato sono posti sul medesimo piano gerarchico-informativo e vanno, pertanto, intesi come due unità nucleari. Ringrazio Filippo Pecorari, del gruppo di ricerca coordinato da Angela Ferrari (Università di Basilea), per il prezioso confronto su questo e su altri esempi esposti nel presente lavoro. 
(10a) // / Veo las luces ahora de la ciudad de Nantes en el desasosiego de esta noche insomne $/$ Nucleo $\mathrm{y}$ de pronto me acuerdo de Alina /Nucleo y me parece descubrir en esa mujer aparentemente frágil el vivo retrato de la madre de Jules Verne, /Nucleo que tenía un nombre que parece casi una corriente de aire: /Appendice // $\mathrm{E1} / /$ / Sophie Allote de la Fuye. /Nucleo // // / También el nombre de Alina tiene algo de brisa fresca $\mathrm{y}$, /Nucleo- aunque no fuera así, /Appendice necesito creer en esto, / /Nucleo necesito confiar en que ella se convierta en mi aliada / Nucleo y sea la corriente de aire que elimine la enfermedad literaria de Montano /Nucleo y de paso, /Appendice- a ser posible, / Appendice la mía. /-Appendice //

(VMa: 23) (10b) // / Vedo ora le luci della città di Nantes nell'inquietudine di questa notte insonne /Nucleo e d'un tratto mi ricordo di Alina/ ${ }^{\text {Nucleo }} \mathrm{e}$ mi sembra di scoprire in quella donna apparentemente fragile il ritratto della madre di Jules Verne, /Nucleo che aveva un nome simile a una folata di vento: /Appendice // $\mathrm{E1} / /$ / Sophie Allote de la Fuye. /Nucleo // $/ / /$ Pure il nome di Alina ha qualcosa della brezza fresca e, / Nucleo- anche se non fosse così, /Appendice ho bisogno di crederci, /-Nucleo ho bisogno di confidare nel fatto che lei diventi mia alleata / Nucleo e sia la folata di vento che eliminerà la sindrome letteraria di Montano /Nucleo e possibilmente, /Appendice- già che ci siamo, /Appendice anche la mia. /-Appendice // $\mathrm{E3}$ (VMb: 18)

Nell'esempio (10), la collocazione incipitaria di enunciato di también potrebbe favorire una lettura come connettivo testuale con valore argomentativo anziché come focalizzatore additivo. Questa interpretazione sarebbe, inoltre, favorita dal traducente italiano pure che si presta, più di anche, a veicolare una connessione tra enunciati con un valore additivo-argomentativo caratterizzato da una sfumatura concessiva (De Cesare 2004a: 23; Ricca 2017: 50). Tuttavia, due fattori rendono questa lettura inadeguata: uno formale, dato dall'assenza di una virgola dopo l'avverbio e un altro semantico, dato dal cotesto sinistro in cui è codificata l'alternativa al fuoco dell'avverbio ("el nombre de Alina"), vale a dire, il nome della madre di Jules Verne: "Sophie Allote de la Fuye". L'individuazione dell'alternativa che si annette al fuoco dell'avverbio sulla base di un termine presupposto comune (cfr. § 4.2: Tab. 1) innesca, così, una lettura come avverbio focalizzante e non come connettivo testuale. Per questa ragione, la resa con il traducente pure, a discapito, ad esempio, di anche, non ha una ragion d'essere funzionale ma va interpretata come una scelta stilistica della traduttrice, probabilmente dovuta alla co-occorrenza della locuzione concessiva «anche se» dell'Appendice successiva.

Diverso è il caso di (11), in cui l'estrazione di también nel Quadro mediante virgola e la natura della connessione veicolata determinano la funzione di connettivo che stabilisce una relazione logica di addizione fra due enunciati e non, quindi, quella di focalizzatore di un sintagma. In effetti, l'attivazione della funzione di connettivo testuale di también è associata proprio alla sua saturazione senza residui, cioè senza altro materiale linguistico, dell'unità di Quadro (De Cesare / Borreguero 2014: 78). In virtù di ciò, l'equivalente italiano scelto dalla traduttrice (11b) non è il focalizzatore anche, ma la locuzione e poi che, al pari di inoltre e in più, veicola una connessione additiva tra due enunciati: 
(11a) // / Al menos durante un tiempo, /Quadro necesito no relacionar nada con la literatura, /Nucleo descansar como sea de ella. /Appendice // E1 // / También, /Quadro aunque sólo sea por un tiempo, /Quadro voy a dejar aparcado este diario que se me estaba volviendo novela. / Nucleo // E2

(VMa: 39) (11b) // / Almeno per un po', /Quadro ho bisogno di non mettere niente in rapporto con la letteratura, ${ }^{\text {Nucleo }}$ di riposare come posso da lei. /Appendice // $/ / /$ / E poi, /Quadro anche se sarà solo per poco tempo, /Quadro lascerò parcheggiato questo diario che mi si stava trasformando in romanzo. $/$ Nucleo $/ /$ E2 (VMb: 31-32)

Si osservi inoltre che la traduzione italiana con e poi (11b) veicola l'attivazione di un valore non solo additivo ma anche temporale del primo Quadro, il che potrebbe favorire un'interpretazione dell'unità successiva ("anche se sarà solo per poco tempo") come un'Appendice posta a specificare il medesimo contenuto semantico, ossia il valore temporale. Questa lettura, tuttavia, non sarebbe applicabile al testo originale (11a), in cui il Quadro in apertura dell' $\mathrm{E}_{2}$ veicola un valore unicamente additivo, non temporale, che impedirebbe dunque un'interpretazione dell'unità successiva come Appendice. Per questa ragione, sommata all'apertura a destra del segmento - vòlto a definire la cornice entro cui interpretare il Nucleo seguente più che a specificare la pertinenza del Quadro a sinistra - considero entrambe le unità due Quadri informativi.

Relativamente alla collocazione di también in co-occorrenza con altro materiale linguistico di origine denotativa, a prevalere - indipendentemente dall'unità: in questo caso (12), il Nucleo di un Inciso che marca discontinuità enunciativa - è quasi sempre la funzione di avverbio focalizzante su quella di connettivo testuale. Nell'esempio (12), infatti, la connessione additiva tra $i$ due enunciati è realizzata dal connettivo che satura il Quadro in apertura del secondo enunciato: además per lo spagnolo e inoltre per l'italiano, entrambi connettivi intrinsecamente testuali. Diversamente, también opera sul sintagma verbale e può, pertanto, essere tradotto con anche $(12 \mathrm{~b})^{14}$ :

(12a) // / Me he convertido en un parásito literario de mí mismo, /Nucleo // // / pues he hallado en mis problemas /Nucleo- tras la publicación de Nada más jamás /Appendice la inspiración para volver al mundo de la creación de ficciones. /-Nucleo // $\mathrm{E}_{2} / / /$ Y además, /Quadro [me dije también, $\left.{ }^{\mathrm{Nucle}}\right]^{\text {Inciso }}$ tal vez esto pueda ayudarme a sanar. Nucleo // E3 $^{\text {NMa: 115-116) }}$ (12b) // / Sono diventato un parassita letterario di me stesso, /Nucleo // trovato nei problemi successivi alla pubblicazione di Mai più niente l'ispirazione per tornare al mondo della creazione di finzioni. /Nucleo // ${ }_{\mathrm{E} 2} / /$ / Inoltre, /Quadro [mi sono anche detto, $\left.{ }^{\text {Nucleo }}\right]^{\text {Inciso }}$ può darsi che questo mi aiuti a guarire. /Nucleo // $\mathrm{E3}(\mathrm{VMb}: 96)$

Anche in (13) también opera come focalizzatore anteposto al sintagma verbale ed è quindi tradotto con anche, post-modificatore del verbo. Si noti pure che l'E $\mathrm{E}_{2}$ presenta una cesura informativa rappresentata da una virgola che non è implicata dalla sintassi ma che, al contrario, svolge un ruolo profondamente comunicativo: isola,

\footnotetext{
4 Si osservi che il secondo enunciato $\left(\mathrm{E}_{2}\right)$ dell'esempio (12) presenta un'organizzazione informativa diversa nelle due lingue, dovuta alla traduzione de-illocutiva del sintagma preposizionale in spagnolo ("tras la publicación") mediante un sintagma aggettivale in italiano ("successivi alla pubblicazione"). L'assenza della virgola prima di tras, d'altra parte, potrebbe favorire pure una lettura linearizzata, come nella versione italiana, priva anch'essa di virgola. Ciononostante, ho optato per questa segmentazione col fine di evidenziare le ripercussioni, sul piano gerarchico-informativo, della diversa resa interlinguistica.
} 
infatti, un Topic complesso e morfo-sintatticamente pesante dal Comment emarginato a destra. Questa configurazione dell' $E_{2}$ in (13) rende plausibili almeno due interpretazioni informative: la prima, più ancorata al valore pragmatico, considererebbe l'enunciato che precede la virgola un Quadro, e quello che la segue un Nucleo; la seconda lettura - per la quale ho optato in virtù dell'eccessivo, e anomalo, peso sintattico-semantico del Quadro rispetto al Nucleo - fa invece prevalere il valore semantico e la relazione di aboutness, e considera, quindi, l'E $\mathrm{E}_{2}$ formato da un unico Nucleo, in cui la virgola posta nel punto di transizione fra Topic e Comment ha un valore formale ( $v s$. funzionale) dovuto al peso morfo-sintattico del cotesto a sinistra del segno interpuntivo:

(13a) // / Después de todo, /Quadro en los diarios íntimos no se dialoga únicamente con uno mismo, $/$ Nucleo también se habla con los demás: / Nucleo // $\mathrm{E} 1 / /$ / todas las conversaciones que en la vida real nunca podemos llevar a término porque acabarían en estallidos de violencia $_{\text {Topic }}$, se van depositando en el diario $_{\text {Comment }}$. Nucleo // ${ }_{\text {E2 }}$ (VMa: 128) (13b) // / Dopotutto, /Quadro nei diari intimi non dialoghiamo unicamente con noi stessi, /Nucleo ma parliamo anche con gli altri: /Nucleo $/ /{ }_{\mathrm{E} 1} / / /$ tutte le conversazioni che nella vita reale non riusciamo mai a portare a termine perché finirebbero in esplosioni di violenzaTopic, vengono depositate nel diario ${ }_{\text {Comment }}$. Nucleo // E2 (VMb: 107)

In altri casi in cui, proprio come in (12) e in (13), también opera come focalizzatore additivo, la decisione di optare per una strategia traduttiva diversa da anche (o pure) risponde, in molti casi (ma cfr. § 4.2) a scelte stilistiche della traduttrice e non a specifiche restrizioni della lingua italiana. Più precisamente, in (14) il valore additivo di también viene convogliato nel verbo aggiungere che sostituisce l'originale decir, semanticamente neutro rispetto al valore additivo. Invece, in (15a), también satura il Nucleo operando come focalizzatore additivo del sintagma verbale implicito, espresso nell' $\mathrm{E}_{1}$ del cotesto anteriore ("se vuelve loco"), mentre nella traduzione (15b) anche si lega al sintagma preposizionale che lo segue ("con l'orfidal") all'interno del Nucleo.

(14a) // / Mi madre habla en el poema de una casa en la calle Provenza, /Nucleo tal vez un deliberado error que obedece a su secreto deseo de cambio de domicilio, /Appendice y yo diría que también de cambio de marido. I Appendice // ${ }_{\mathrm{E}}$ (VMa: 129)

(15a) // / Luego ve la televisión y se vuelve loco si no toma el orfidal. /Nucleo // // / Pero si lo toma, /Quadro también, /Nucleo / sólo que tomándose las cosas con más calma, Nucleo aunque sin dejar de percibir la grisura de su existencia de escritor atado de por vida a su oficio y la monotonía de su tragedia cotidiana. /Appendice // E2 (VMa: 140) (14b) // / Mia madre nella poesia parla di una casa in calle Provenza, /Nucleo forse un deliberato errore che risponde al suo segreto desiderio di cambiare dimora, /Appendice e io aggiungerei di cambiare marito. /Appendice $/ /_{\mathrm{E}}$ (VMb: 107)

(15b) // / Poi guarda la televisione e impazzisce se non prende l'orfidal. /Nucleo // $/ / /$ In realtà gli succede anche con l'orfidal, /Nucleo solo che così prende le cose con più calma, /Nucleo sebbene non smetta di percepire il grigiore della sua esistenza di scrittore legato vita natural durante al suo mestiere e alla monotonia della sua tragedia quotidiana. / Appendice // 2 (VMb: 117) 
La scelta di riformulare la prima parte dell' $\mathrm{E}_{2}$ in (15b) è senz' altro dettata dalla volontà della traduttrice di rendere meno ambigua la sequenza che, nel testo originale, è caratterizzata da una sintassi tortuosa e poco trasparente. Inoltre, la saturazione del Nucleo da parte di también è giustificata dalla sua maggiore autonomia testuale rispetto ad anche: autonomia che si traduce nella possibilità di un aggancio a distanza con il sintagma verbale dell' $\mathrm{E}_{1}$. La medesima configurazione informativa si sarebbe potuta mantenere in italiano optando per il traducente pure, che gode di maggiore autonomia testuale rispetto ad anche. Quando, come in (15a), también satura un'UI, i suoi effetti semantici e la sua portata dipendono, in buona misura, dal tipo di UI a cui si aggancia. A tal proposito, De Cesare / Borreguero (2014: 84) osservano che quando una particella discorsiva satura un'Appendice che si lega a un Nucleo, la sua funzione può essere sia quella di focalizzatore sia quella di connettivo testuale. Nell'esempio (16) también satura proprio un'Appendice interposta a un Nucleo, tuttavia, la prossimità al verbo nell'immediato cotesto anteriore conferisce all'avverbio uno statuto ambiguo, a metà strada fra focalizzatore del verbo e connettivo testuale:

(16a) // / «De adolescente, /Quadro leyendo a Borges», /Quadro [me dijo Aira, $]^{\text {Inciso } ~ « v i ~ d o ́ n d e ~}$ estaba la esencia de la literatura. / Nucleo // $/ / /$ / Eso fue definitivo, ${ }^{\text {Nucleo }}$ pero después descubrí, /Nucleo- también, /Appendice que la literatura no tiene una esencia, $/{ }^{- \text {Nucleo }}$ sino muchas históricas y contingentes. /Nucleo // $/ /$ / Así que / Quadro fue fácil escapar de la órbita borgiana, /Nucleo tan fácil como volver, ${ }^{\text {Nucleo }}$ o como no haber escapado nunca. /Nucleo // ${ }_{\mathrm{E} 3}$ (VMa: 77) (16b) // / "Da adolescente, /Quadro leggendo Borges," /Quadro [mi disse Aira, ] ${ }^{\text {Inciso "vidi }}$ dove stava l'essenza della letteratura. /Nucleo // $\mathrm{E1} / / / \mathrm{Fu}$ qualcosa di definitivo, /Nucleo ma poi scoprii, /Nucleo- pure, /Appendice che la letteratura non ha un'essenza sola, $/{ }^{- \text {-Nucleo }}$ ma molte, /Nucleo storiche e contingenti. /Appendice // // / Così, /Quadro fu facile scappare dall'orbita borgesiana, /Nucleo tanto facile come tornarvi, ${ }^{\text {Nucleo }}$ o come non esserne mai scappato. Nucleo $^{\text {Nat }}$ // ${ }_{\mathrm{E} 3}(\mathrm{VMb}: 64)$

In effetti, nell'esempio (16), a favorire una lettura di también come focalizzatore interverrebbero due fattori: la prossimità al verbo descubrir e la traducibilità con anche. D'altra parte, però, sia l'altrettanto valida traducibilità con inoltre sia la sostituibilità, nel testo originale, con además - che, come si è visto, sono entrambi connettivi intrinsecamente testuali - sosterrebbero una interpretazione di también, e del suo traducente pure, come connettivi testuali. Inoltre, la delimitazione dell'Appendice tra due virgole dà origine a un fenomeno di discontinuità informativa più tipicamente associato alla funzione connettiva che a quella focalizzante. Permane, in definitiva, un certo grado di ambiguità circa la portata dell'avverbio; ambiguità di cui è sintomatica la scelta del traducente italiano pure a discapito degli altrettanto ammissibili anche e inoltre che, tuttavia, non avrebbero mantenuto l'ambiguità originale, al contrario, avrebbero indotto, rispettivamente, l'attivazione della funzione di focalizzatore e quella di connettivo. Si osservi, inoltre, che la virgola inserita dalla traduttrice nell' $E_{2}(16 b)$ - e assente nel testo originale (16a) - tra il quantificatore ("molte") e i qualificativi ("storiche e contingenti") ha un valore fortemente comunicativo, vòlto a conferire rilievo informativo ai due elementi a destra del segno. Da ciò deriva una diversa segmentazione del testo in spagnolo (un Nucleo) e in italiano (un Nucleo e un'Appendice). 
Nel testo (17) è presente anche una catena di particelle discorsive ${ }^{15}$ composta da tre particelle con valore additivo:

(17a) // / Su pálida frente es un mapa confuso. /Nucleo $/ /{ }_{E 1} / /$ / También esto me pareció suave / ${ }^{\text {Nucleo }} \mathrm{y}$ además dulzón /Appendice y hasta almibaradamente poético. /Appendice $/ /_{\mathrm{E} 2}$ (VMa: 150) (17b) // / La sua pallida fronte è una mappa confusa. / Nucleo // // / Anche questo mi è sembrato innocuo / Nucleo e anche sdolcinato / Appendice e per giunta mielosamente poetico. / Appendice // (VMb: 126)

In (17b), il crescendo di particelle additive che introducono ciascun aggettivo («innocuo», «sdolcinato» e «poetico») qualificando l' $\mathrm{E}_{1}$ non è stato reso in italiano in modo del tutto fedele. Innanzitutto, non viene rispettata la variatio del testo originale, che ricorre a tre diversi lessemi (también, además e hasta) che in italiano diventano soltanto due (anche e per giunta). Inoltre, l'ordine con cui sono disposti también, además e hasta in (17a) configura un climax ascendente che non è mantenuto, se non parzialmente, nella traduzione. Infatti, se da una parte il traducente per giunta restituisce la scala additiva culminativa proiettata da hasta, dall'altra, la seconda occorrenza di anche non contiene, dell'originale además, né il valore tipicamente argomentativo (Santos Río 2003: 175) né quello - sia pur determinato contestualmente - scalare (Montolío 2001: 141; Fuentes 2009: 36). Una traduzione più attenta ai valori semantici veicolati dalle particelle potrebbe essere la seguente: "Anche questo mi è sembrato innocuo oltre che sdolcinato e per giunta mielosamente poetico."

\subsection{También con fuoco a sinistra}

Come si è visto (§ 2.1), lo sp. también è dotato di una grande mobilità posizionale: può infatti precedere o seguire, linearmente o a distanza, il suo fuoco, indipendentemente dalla categoria grammaticale di questo (Kovacci 1999: 774). Diversamente, l'it. anche ha una configurazione più ristretta e tende, in contesti non marcati, a seguire un fuoco verbale e a precederne uno nominale (De Cesare 2015: 37). Inoltre, tale principio del fuoco a destra fa sì che anche tenda a proiettare il suo fuoco sul cotesto successivo, a meno che non sia seguito da una forma verbale. Di conseguen$\mathrm{za}$, anche può essere post-modificatore solo quando non è seguito da elementi linguistici con cui potrebbe legarsi. Tipicamente, dunque, il focalizzatore italiano può essere post-modificatore solo quando nell'immediato cotesto successivo è presente una forma verbale, la quale non innesca una lettura ambigua dal momento che anche tende a occupare la posizione post-verbale e non quella preverbale.

Il diverso comportamento sintattico di también - caratterizzato da ampia mobilità - e di anche - che obbedisce al principio del fuoco a destra - diventa particolarmente problematico, in un'ottica contrastiva, quando il fuoco di también è posto a sinistra del focalizzatore. In (18), per esempio, l'istruzione semantica di uguaglianza veicolata da también, il cui fuoco ("la literatura") è espresso a sinistra, viene resa in modo improprio dal traducente anche che, proiettando il fuoco a destra, aggiunge una comparazione:

\footnotetext{
Bazzanella (2001: 45) attua una distinzione fra le "catene" e i "cumuli" di segnali discorsivi (SD): «cumuli, intesi come sequenza di più SD in cui ognuno svolge la stessa funzione, tipicamente come fatismi [...] catene, intese come sequenza di più SD in cui ognuno svolge una funzione specifica».
} 
(18a) «Al tacto el pabellón de mi oreja se notaba fresco, áspero, frío y jugoso, como una hoja de árbol», escribe Kafka en su diario de 1910. Su frase me lleva a otra, me lleva al recuerdo de una que le escuché a Claudio Magris, una noche en Barcelona: // / «Puede que la literatura ${ }_{\text {fuoco }}$ sea también parte del mundo del modo que lo son, $/$ Nucleo- por ejemplo, /Appendice las hojas.// $/$-Nucleo $/ /$ E La frase de Magris no sólo me consuela sino que me devuelve al mundo. Literatura y mundo entran en armonía. (VMa: 157) (18b) "Al tatto il padiglione del mio orecchio era fresco, ruvido, freddo, succoso come una foglia d'albero" scrive Kafka nel suo diario del 1910. La sua frase mi porta a un'altra, mi porta il ricordo di una frase che ho sentito dire a Claudio Magris, una notte a Barcellona: // / "Può darsi che la letteratura sia anche parte del mondo nel modo in cui lo sono, / Nucleo- per esempio, /Appendice le foglie." /-Nucleo $/ /$ Ea frase di Magris non solo mi consola ma mi restituisce al mondo. Letteratura e mondo entrano in armonia. (VMb: 132)

In effetti, in (18b) la traduzione con anche suggerisce una lettura additiva, nel senso che "la letteratura sia parte di $x$ cose e, tra queste, del mondo", tuttavia, l'immediato cotesto seguente aiuta retroattivamente a disambiguare. Si tratta, insomma, di un'interpretazione che tradisce il senso dell'originale, vòlto a esprimere una relazione di uguaglianza (la letteratura / mondo $=$ le foglie / mondo) che si sarebbe potuta restituire in modo più univoco con il traducente a sua volta oppure mediante la ripresa pronominale dopo anche: "può darsi che la letteratura sia anch'essa parte del mondo [...]".

Così, in assenza di ripresa pronominale dopo l'avverbio, anche proietta il fuoco a destra, aprendo la comparazione. Per questo, ove también veicoli un valore semantico di uguaglianza (A come $\mathrm{B})$ e non di addizione $(\mathrm{A}+\mathrm{B})$, una strategia traduttiva che consenta di esprimere il medesimo stato di cose consiste nel ricorso alla locuzione $a$ sua/mia volta, come negli esempi (19) e (20):

(19a) // / Llevado también yo por cierta voluntad de servicio, /Quadro quisiera decirle al lector que, /Nucleo- salvando las insalvables distancias, /Appendice mi modus operandi puede recordar, $/$-Nucleo- aunque no caí en la cuenta hasta hace poco /Appendice $[-$ hasta que leí Segunda mano -, $]^{\text {Inciso }}$ al de Borges. $/$-Nucleo $/ / \mathrm{E}$ (VMa: 121)

(20a) // / Y poco después, /Quadro con su mano izquierda, /Quadro que era la que le quedaba libre /Appendice [- porque con la derecha volvía a llamar al camarero -, $]^{\text {Inciso }}$ se tocó su monstruosa oreja derecha. /Nucleo // $/ / /$ Me acordé de Gombrowicz: /Nucleo // ${ }_{\mathrm{E} 2}^{\mathrm{E} / / /}$ / $\mathrm{Si}$ queréis expresar que os gustó mi obra, /Quadro tocad sencillamente, / $/$ ucleo- al verme, /Appendice vuestra oreja derecha.»/ /-Nucleo // $\mathrm{E}_{3} / / /$ - Girondo - /Nucleo // $\mathrm{E} 4 / /$ / dije, /Nucleo tocándome también la oreja - . /Appendice $/{ }_{\mathrm{E} 5}$ (VMa: 151-152) (19b) // / Spinto a mia volta da una certa volontà di servire, /Quadro vorrei dire al lettore che, /Nucleo- salvando le insalvabili distanze, / Appendice il mio modus operandi può talvolta ricordare, /-Nucleo- sebbene non me ne fossi accorto fino a poco fa/Appendice [- finché non ho letto Segunda mano -, $]^{\text {Inciso }}$ quello di Borges. /-Nucleo // $(\mathrm{VMb}: 101)$

(20b) // / E poco dopo, /Quadro con la mano sinistra, /Quadro che era quella rimasta libera /Appendice [- perché con la destra stava richiamando il cameriere -, $]^{\text {Inciso }}$ si è toccato il mostruoso orecchio destro. /Nucleo // $/ / / /$ Mi sono ricordato di Gombrowicz: /Nucleo // // / "Se volete farmi capire che l'opera vi è piaciuta toccatevi l'orecchio destro." /Nucleo // E3 // / "Girondo" /Nucleo // E4 // / ho detto, /Nucleo toccandomi a mia volta l'orecchio. /Appendice // $(\mathrm{VMb}: 127)$ 
Gli esempi (18), (19) e (20) appena visti dimostrano che la segmentazione del $\mathrm{MB}$ non consente di predire il tipo di relazione veicolata (addizione vs uguaglianza), la cui identificazione dipende unicamente dalla semantica del cotesto. Infatti, i tre esempi presentano también come indicatore di uguaglianza in tre diverse UI, rispettivamente: Nucleo (18), Quadro (19) e Appendice (20). Tuttavia, è possibile ricavare un dato: también marca la relazione di uguaglianza in modo più trasparente di anche che, invece, marca convenzionalmente un'interpretazione additiva. Affinché il valore di uguaglianza veicolato da también sia mantenuto nella resa in italiano, gli esempi analizzati suggeriscono di ricorrere ad altre strategie traduttive, come la locuzione a mia/sua/loro volta, oppure di codificare, mediante ripresa pronominale dopo l'avverbio, il referente che costituisce il fuoco di anche e sul quale il focalizzatore opera come indicatore di uguaglianza.

Oltre a veicolare una relazione di uguaglianza, le occorrenze di también negli esempi (18) e (20) sono accomunate da un altro fattore, che risulta cruciale in una prospettiva contrastiva con l'italiano: il fuoco di también è posto a sinistra dell'avverbio. L'ambiguità referenziale innescata dall'ampia mobilità distribuzionale di también deve essere risolta in modo da veicolare la stessa relazione e proiettare lo stesso insieme di elementi in gioco nella traduzione in italiano. Gli avverbi della lingua italiana, infatti, hanno una configurazione più locale che richiede lo scioglimento dell'ambiguità superficiale, ammessa invece dalla lingua spagnola.

Innanzitutto, dunque, occorre identificare il fuoco dell'avverbio mediante la ricostruzione dello schema comparativo soggiacente. Infatti, gli avverbi focalizzanti - talvolta denominati, proprio in virtù di questa proprietà, "avverbi paradigmatizzanti” (Nølke 1983; De Cesare 2004: 215; Ferrari et al. 2008: 340) - proiettano una struttura comparativa ${ }^{16}$ in cui un termine presupposto funge da sfondo comune a dei termini comparati: il paradigma di alternative e il fuoco dell'avverbio. Vi sono degli indizi per identificare il paradigma di alternative che si annette al fuoco dell'avverbio: innanzitutto, fuoco e alternativa sono caratterizzati da un parallelismo strutturale e concettuale (Perrin-Naffakh 1996: 139). Nello specifico, l'alternativa e il fuoco dell'avverbio hanno la stessa forma e funzione sintattica (Andorno / De Cesare 2017: 160) e, talvolta, presentano anche dei parallelismi semantici e/o lessicali, ad esempio, mediante iperonimi o nomi collettivi (Andorno / De Cesare 2017: 178). Il termine presupposto, invece, deve essere semanticamente compatibile con il fuoco e il paradigma di alternative (Andorno / De Cesare 2017: 160).

In altre parole, la struttura comparativa proiettata dagli avverbi focalizzanti può essere ricostruita seguendo i parallelismi sintattici e semantico-lessicali che intercorrono fra i suoi termini. Più precisamente, i termini comparati (fuoco dell'avverbio e paradigma di alternative) presentano la medesima struttura sintattica e, talvolta, anche parallelismi sul piano semantico e lessicale; il termine presupposto, invece, è identificabile sulla base di una compatibilità semantica con le proposizioni in cui compaiono i termini comparati. La struttura comparativa proiettata dagli avverbi focalizzanti ${ }^{17}$ è schematizzabile nel modo seguente (Tabella 1):

16 Nel presente lavoro ricorro alla terminologia di Claudia Borzi (comunicazione personale in data 6 giugno 2019), per cui la "proposizione presupposta e nota" (in base alla terminologia di Ferrari et al. 2008: 357) che funge da sfondo comune è denominata "termine presupposto", mentre la "variabile aperta" (Ferrari et al. 2008: 342) consiste nei "termini comparati", ossia "alternativa" e "fuoco" dell'avverbio.

17 L'identificazione della struttura comparativa soggiacente è un criterio di analisi pertinente anche per stabilire l'ambito operativo dei connettivi testuali. A differenza degli avverbi focalizzanti, però, i connettivi testuali 


\begin{tabular}{|l|l|}
\hline \multicolumn{2}{|l|}{ Termine presupposto } \\
\hline \multirow{2}{*}{ Termini comparati } & Alternativa \\
\cline { 2 - 2 } & Fuoco dell'avverbio \\
\hline
\end{tabular}

Tabella 1. Struttura comparativa proiettata dagli avverbi focalizzanti

Così, nell'esempio (21), nonostante la contiguità rispetto alla forma verbale («haya envejecido»), il fuoco di también non è costituito dal verbo, cosa che ha delle ripercussioni sulla scelta e sulla posizione del traducente in italiano. Esempi come questo (21), peraltro, contraddicono in parte le indicazioni date dalla grammatica, secondo la quale, nella posizione intercalata fra i componenti del gruppo verbale, la portata del focalizzatore: «no podría sobrepasar el ámbito de dicho segmento» (NGLE 2009: 2992). Tuttavia, in (21), sebbene también sia collocato all'interno della forma verbale, più precisamente, tra l'ausiliare e il participio - collocazione peraltro rara in spagnolo -, il suo fuoco è costituito da «la literatura», su cui opera retroattivamente e la cui alternativa è data dalla prima persona singolare, espressa nell'enunciato che inaugura l'esempio. Lo schema comparativo proiettato dal focalizzatore, e che consente, dunque, di determinare il suo fuoco è il seguente: "envejecer" (termine presupposto); "yo" (alternativa); "la literatura" (fuoco dell'avverbio). Si considera, pertanto, soddisfacente la resa in italiano (21b) mediante la locuzione a sua volta che consente un aggancio trasparente al referente «la letteratura»; infatti, una resa con anche avrebbe spostato il fuoco dell'avverbio sul participio contiguo («invecchiata»), promuovendo il termine presupposto a termine comparato:

(21a) Cuando fui a Nantes, en noviembre del año pasado, yo aún no había envejecido veinte años de golpe en una sola tarde en Budapest. // / Y la literatura fuoco $_{\text {andaba mal, }}$ /Nucleo pero no tanto como ahora, / Nucleo que no es ya que haya también envejecido mucho, Nucleo es que parece el imperio austrohúngaro precipitándose a su destrucción. / Nucleo $/ / \mathrm{E}$ (VMa: 246) (21b) Quando sono stato a Nantes, a novembre dell'anno scorso, non ero ancora invecchiato di vent'anni tutti d'un colpo in una sola sera a Budapest. // / E la letteratura ${ }_{\text {fuoco }}$ se la passava male, /Nucleo ma non tanto come adesso, /Nucleo che non solo è a sua volta molto invecchiata, /Nucleo ma somiglia sempre di più all'Impero austro-ungarico mentre precipita verso la distruzione. /Nucleo // $\mathrm{E}$ VMb: 210)

La ricostruzione della struttura comparativa proiettata dal focalizzatore è dunque cruciale in un'ottica contrastiva con l'italiano, specie quando il fuoco dell'avverbio in spagnolo è posto a sinistra. Il fuoco di también può trovarsi a sinistra e anche molto a distanza, come in (22a) dove tra il focalizzatore e il suo fuoco ("Rosario Girondo") ci sono un'Appendice e un Inciso. Diversamente, anche focalizza il termine alla sua destra e per questo, nella traduzione in italiano (22b), l'assetto sintagmatico dell'enunciato è riorganizzato affinché l'avverbio sia posto a ridosso del fuoco come

proiettano uno schema argomentativo in base al quale il termine presupposto è dato dalla tesi (o conclusione), espressa o tacita, su cui si basano gli argomenti esposti, ossia i termini comparati: quello introdotto dal connettivo e quello, espresso o tacito, delle alternative. Non si considerano, invece, connettivi i cosiddetti "estructuradores de la información" (Martín Zorraquino/Portolés 1999: 4080), come en primer lugar, por un lado, por último ecc., che si occupano della disposizione spaziale degli enunciati nel testo senza incidere sull'assetto logico-semantico e che, pertanto, non proiettano uno schema di tipo argomentativo. 
pre-modificatore lineare. Così, grazie all'anteposizione del focalizzatore rispetto al suo fuoco, viene rispettata la struttura comparativa soggiacente: "ser un fantasma ambulante" (termine presupposto), "quien es capaz de mirar de esa manera" (alternativa), "Rosario Girondo" (fuoco dell'avverbio):

(22a) Yo creo ver ahora mismo que quien es capaz de mirar de esa manera no está demasiado agarrado a la materialidad de la vida y es un fantasma ambulante. // / Rosario Girondo $_{\text {fuoco }}$, /Quadro por ejemplo /Appendice $[-$ yo, no mi madre - ], ${ }^{\text {Inciso }}$ es también un fantasma ambulante, ${ }^{\text {Nucleo }}$ se pasea por estas páginas tratando de aprender a saber leer a los demás, tratando de estar fuera de sí y mirar, / Nucleo porque aspira a mirar algún día como miraba Soares o a leer como leía Pessoa, /Nucleo que nunca leyó un libro entregándose a él ${ }^{\text {Nucleo }}[\text { - salvo si era de Soares -, }]^{\text {Inciso }}$ porque siempre a cada paso la memoria [- como me ocurría a mí anteayer leyendo a Michaux -] Inciso le interrumpía la secuencia narrativa: / Nucleo // «Después de unos minutos, quien escribía era yo, y lo que estaba escrito no estaba en ninguna parte.» Una elegante forma de decir que su Yo hacía suyo lo que estaba fuera de él. Es lo que trata de imitar mi Yo desde hace un tiempo. Ventana no me falta. (VMa: 183-184) (22b) Credo di capire proprio adesso che chi è capace di guardare in quel modo non è troppo ancorato alla materialità della vita ed è un fantasma ambulante. // / Anche Rosario Girondo ${ }_{\text {fuoco }}$, /Quadro per esempio /Appendice [- io, non mia madre - $]^{\text {Inciso è un fantasma }}$ ambulante, /Nucleo passeggia per queste pagine cercando di imparare a saper leggere gli altri, provando a stare fuori di sé e guardare, /Nucleo perché aspira a guardare un giorno come guardava Soares o a leggere come leggeva Pessoa, $/^{\text {Nucleo }}$ che non lesse un solo libri abbandonandovisi / Nucleo [- a meno che non fosse di Soares -, $]^{\text {Inciso }}$ perché sempre, /Nucleoa ogni passo, /Appendice la memoria [- come succedeva a me l'altro ieri nel leggere Michaux - $]^{\text {Inciso }}$ gli faceva perdere, / Nucleo- interrompendolo, /Appendice il filo narrativo: /-Nucleo // "Dopo qualche minuto, a scrivere ero io, e quello che era scritto non era da nessuna parte". Un'elegante maniera di dire che l'Io faceva suo ciò che stava fuori di sé. È ciò che cerca di imitare il mio Io da un po' di tempo a questa parte. Finestre non me ne mancano. (VMb: 154)

Il problematico aggancio anaforico dei focalizzatori dell'italiano anche e pure si manifesta non soltanto nei casi come quello appena visto (22b), in cui il fuoco dell'avverbio è collocato a sinistra e a distanza, ma anche quando esso precede linearmente un focalizzatore che sia seguito da elementi linguistici sui quali potrebbe proiettare i suoi effetti, caratteristicamente, dunque, qualunque forma linguistica non verbale. Ad esempio, in (23a) también opera anaforicamente e linearmente su "Montano" mentre, nella traduzione (23b), pure opera linearmente a destra sul pronome "lui", che riprende anaforicamente il fuoco dell'avverbio. È la ripresa pronominale a consentire di mantenere inalterata la struttura comparativa soggiacente: "estar enfermo de literatura" (termine presupposto), "yo" (alternativa), "Montano" (fuoco dell'avverbio). Senza il pronome "lui" dopo l'avverbio pure, in effetti, si avrebbe una lettura ambigua in base alla quale il termine presupposto potrebbe essere interpretato come termine comparato, ossia come fuoco dell'avverbio: "con Montano pure [malato di letteratura $]_{\text {fuoco }}$ ". 
(23a) Quizá tenía razón Rosa cuando me dijo que elegir Nantes [- con Montano fuoco $_{\text {f }}$ enfermo también, / Nucleo- aunque con distinta fiebre, /Appendice de literatura /-Nucleo -$]^{\text {Inciso }}$ no iba a ser precisamente la solución más adecuada para que durante unos días yo pudiera descansar de mis temidas críticas y de mi obsesión enfermiza por los libros y de mi manía de verlo todo desde la literatura. (VMa: 21-22) (23b) Forse aveva ragione Rosa a dirmi che scegliere Nantes [- con Montano pure lui $_{\text {fuoco }}$ malato di letteratura, /Nucleo anche se con una febbre diversa /Appendice -$]^{\text {Inciso }}$ non sarebbe stata esattamente la soluzione più adeguata per potermi riposare qualche giorno dalle mie temute critiche e dalla malsana ossessione per il libri, nonché dalla mania di vedere tutto dal punto di vista della letteratura. (VMb: 16-17)

Lo stesso fenomeno si produce nell'esempio (24a), dove il fuoco «mi hijo» è contiguo, collocato nell'immediato cotesto anteriore di también, tuttavia, nella resa in italiano (24b) si rende necessaria la ripresa pronominale con «lui» dopo anche, così da mantenere la medesima struttura comparativa in cui il termine presupposto è dato da "herido por las letras", l'alternativa è "yo" e il fuoco "mi hijo".

(24a) Rosa me dijo que yo necesitaba un viaje urgente, cambiar mi exagerada respiración literaria por paisajes y canciones, hacer turismo no cultural, desintoxicarme de mi absorbente labor de crítico, dedicarme a la serena contemplación de la Madre Naturaleza - «mirar con calma cómo nacen, por ejemplo, los tomates en el campo», me dijo textualmente-, observar puestas de sol y pensar en ella, pensar más en ella, que no podía acompañarme en el viaje - por motivos de trabajo -, pero eso, pensar mucho más en ella. // / Pero Rosa también me dijo que no fuera a Nantes, /Nucleo / donde mi hijofuoco $/$ Appendice- $[/ /$ - también herido por las letras, / Nucleo aunque por motivos distintos a los míos $\left./{ }^{A \text { ppendice }}-/ /\right]^{\text {Inciso }}$ podía agravar aún más mi enfermedad. /-Appendice $/ /{ }_{\mathrm{E}}$ (VMa: 22) (24b) Rosa mi ha detto che avevo bisogno urgente di un viaggio, di sostituire la mia esagerata respirazione letteraria con paesaggi e canzoni, di fare turismo non culturale, disintossicarmi dal mio gravoso lavoro di critico, dedicarmi alla serena contemplazione di Madre Natura - "guardare con calma come nascono, per esempio, i pomodori nei campi", testuali parole -, osservare i tramonti e pensare a lei, pensare di più a lei, che non poteva accompagnarmi nel viaggio - per motivi di lavoro -, però sì, pensare molto di più a lei. // / Ma Rosa mi ha anche detto di non andare a Nantes, Nucleo $^{\text {dove mio }}$ figlio /Appendice- $\left[/ /-\right.$ anche lui ${ }_{\text {fuoco }}$ ferito dalle lettere, ${ }^{\text {Nucleo }}$ sebbene per motivi diversi dai miei /Appendice $-/ /]^{\text {Inciso }}$ poteva aggravare ancor più la mia malattia. /-Appendice $/ /{ }_{\mathrm{E}}$ (VMb: 17)

Mediante la ripresa pronominale (24b), anche recupera anaforicamente "figlio" e lo introduce in uno schema comparativo con "padre". Omettendo il pronome "lui", infatti, l'avverbio italiano aggiungerebbe una comparazione proiettando un diverso insieme di elementi in gioco, dove "ferito dalle lettere" costituirebbe non più informazione presupposta e nota bensì nuova. Senza la ripresa pronominale, dunque, anche creerebbe un Fuoco di enunciato. In altre parole, l'assenza di ripresa pronominale apre la comparazione: l'elemento introdotto da anche diventa nuovo e costituisce il fuoco dell'avverbio, non la presupposizione, come avviene invece nel testo in spagnolo (24a) dove también introduce linearmente la clausola presupposta mentre opera retroattivamente sul fuoco a sinistra («mi hijo»). L'ambito di significato della comparazione in italiano è dunque vincolato all'esplicitazione del referente mediante la ripresa pronominale; in sostanza, il pronome ridefinisce la portata della predicazione poiché senza di esso cambia il significato del discorso. 
In breve, a differenza di también, che si fa carico sia del termine presupposto che dei termini comparati, anche non si fa carico del termine presupposto e crea alla sua destra un nuovo Fuoco, dunque un termine comparato. Per questa ragione, e in virtù del principio del fuoco a destra (De Cesare 2015: 37; cfr. § 2.1), anche ha bisogno di esplicitare il proprio referente topicale a destra, altrimenti l'elemento che segue l'avverbio viene interpretato come Comment, come informazione nuova e non più presupposta. La spiegazione potrebbe essere che anche, diversamente da también, ha bisogno di recuperare un'intera clausola; si tratterebbe, così, di un diverso limite dell'ellissi tra i focalizzatori delle due lingue.

Di seguito (Tabella 2), uno schema riassuntivo ${ }^{18}$ della presente analisi contrastiva di también e dei suoi traducenti in italiano sulla base delle funzioni svolte:

\begin{tabular}{|c|c|c|c|c|}
\hline $\begin{array}{l}\text { Funzione } \\
\text { discorsiva }\end{array}$ & $\begin{array}{c}\text { Unità } \\
\text { Informativa }\end{array}$ & $\begin{array}{l}\text { Con altri } \\
\text { elementi }\end{array}$ & $\begin{array}{c}\text { Occorrenza } \\
\text { di también }\end{array}$ & $\begin{array}{c}\text { Equivalenti } \\
\text { in italiano }\end{array}$ \\
\hline
\end{tabular}

\begin{tabular}{|c|c|c|c|c|}
\hline \multirow{3}{*}{$\begin{array}{l}\text { AVVERBIO } \\
\text { FOCALIZZANTE }\end{array}$} & Nucleo & Sì & $\begin{array}{l}\text { Llevado también yo por } \\
\text { cierta voluntad de servi- } \\
\text { cio, quisiera decirle que } \\
{[\ldots]}\end{array}$ & $\begin{array}{l}\text { A mia } \\
\text { volta* } \\
\text { Anche* } \\
\text { Pure* }\end{array}$ \\
\hline & Quadro & Sì & $\begin{array}{l}\text { Pero es también una } \\
\text { metáfora perfecta de la } \\
\text { experiencia literaria. }\end{array}$ & $\begin{array}{l}\text { Anche* } \\
\text { A mia } \\
\text { volta* } \\
\text { Pure }\end{array}$ \\
\hline & Appendice & Sì & $\begin{array}{l}\text { Y además, me dije tam- } \\
\text { bién, tal vez esto pueda } \\
\text { ayudarme a sanar. }\end{array}$ & $\begin{array}{l}\text { Anche* } \\
\text { Pure* }\end{array}$ \\
\hline \multirow{2}{*}{$\begin{array}{l}\text { CONNETTIVO } \\
\text { TESTUALE }\end{array}$} & Quadro & No & $\begin{array}{l}\text { También, aunque sólo sea } \\
\text { por un tiempo, voy a dejar } \\
\text { aparcado este diario. }\end{array}$ & $\begin{array}{l}\text { E poi* } \\
\text { Inoltre } \\
\text { In più } \\
\text { In aggiunta }\end{array}$ \\
\hline & Appendice & No & $\begin{array}{l}\text { Pero después descubrí, } \\
\text { también, que la literatura } \\
\text { no tiene una esencia. }\end{array}$ & $\begin{array}{l}\text { Anche* } \\
\text { Pure* } \\
\text { Inoltre } \\
\text { In più } \\
\text { In aggiunta }\end{array}$ \\
\hline
\end{tabular}

Tabella 2.Schema riassuntivo della traduzione in italiano di también in base alle funzioni svolte

\footnotetext{
18 Nell'ultima colonna, relativa agli equivalenti in italiano, sono seguiti da asterisco $(*)$ i traducenti effettivamente trovati nel testo, mentre sono riportati senza marche grafiche quelli ricavati da altri studi sulla resa di también in italiano: Sainz (2006), Borreguero (2011) e De Cesare / Borreguero (2014). Lo schema riassuntivo qui proposto è una riproduzione parziale di quello messo a punto da Borreguero (2014) per l'avverbio focalizzante sp. incluso, dal quale differisce, sostanzialmente, per la colonna di partenza: Unità Informativa in Borreguero (2014: 50) e funzione discorsiva nel presente studio.
} 


\section{Conclusioni}

Nel presente studio contrastivo basato su un testo parallelo spagnolo-italiano, ho preso in considerazione i seguenti criteri di analisi per la resa in italiano dell'avverbio focalizzante sp. también: (i) l'istruzione semantica veicolata, derivante dal processo di grammaticalizzazione che investe, da una parte, también e, dall'altra, i suoi possibili traducenti in italiano: anche, pure ecc.; (ii) le restrizioni morfosintattiche, maggiori per l'italiano, in quanto lingua dalla configurazione più ristretta rispetto allo spagnolo; (iii) la manifestazione nelle Unità Informative in base al Modello di Basilea, col fine di orientarsi tra le funzioni come avverbio focalizzante e come connettivo testuale, le quali innescano la selezione di traducenti diversi in italiano.

La segmentazione del MB non consente, invece, di predire il tipo di istruzione semantica veicolata (additiva, inclusiva, scalare, di uguaglianza ecc.), la cui identificazione dipende unicamente dalla semantica del cotesto e non dall'occorrenza nelle UI. D'altra parte, l'analisi di testi paralleli mediante il MB permette di evidenziare le ripercussioni sul piano informativo delle diverse scelte traduttive, che comportano - ad esempio, tramite l'uso comunicativo dell'interpunzione - diversi gradi di forza illocutiva, e una diversa distribuzione del peso e del rilievo informativo degli enunciati. Questioni che, da un'ottica traduttiva, si collocano nell'ambito della "fedeltà" - nel senso di un'equivalenza funzionale - della traduzione rispetto al testo di partenza.

Relativamente all'istruzione semantica veicolata, è però possibile ricavare un dato: también marca la relazione di uguaglianza in modo più trasparente di anche che, invece, marca convenzionalmente un'interpretazione additiva ed è, inoltre, maggiormente compatibile con una lettura scalare. Affinché il valore di uguaglianza veicolato da también sia mantenuto nella resa in italiano, gli esempi analizzati suggeriscono di ricorrere ad altre strategie traduttive, come la locuzione a mia/sua/loro volta, oppure di codificare, mediante ripresa pronominale dopo l'avverbio, il referente che costituisce il fuoco di anche e sul quale l'avverbio opera come indicatore di uguaglianza.

Rispetto al secondo criterio, quello morfosintattico, la sua pertinenza in ottica contrastiva fra le due lingue si manifesta caratteristicamente nel caso in cui il fuoco di también è posto a sinistra, linearmente o a distanza, rispetto all'avverbio, il quale sia poi seguito da materiale linguistico su cui potrebbe estendere la propria semantica relazionale, alterando così la struttura comparativa soggiacente e proiettando un diverso insieme di elementi in gioco. Infatti, mentre lo sp. también può, a un livello superficiale, associarsi con vari elementi del cotesto linguistico, i traducenti it. anche e pure tendono a proiettare un fuoco a destra, eccetto quando sono seguiti da un verbo di modo finito, rispetto al quale tendono a operare piuttosto come post-modificatori.

Così, también può operare invariabilmente sul nome, sul verbo e/o sul complemento: Stella también lee Shakespeare; diversamente, in italiano, la ripresa pronominale dopo l'avverbio è necessaria per non spostare sul complemento del verbo il fuoco di anche: Stella legge anche le $i_{\text {fuco }}$ Shakespeare. In effetti, in base al principio del fuoco a destra, in assenza di ripresa pronominale: Stella ${ }_{\text {Topic }}$ legge anche Shakespeare $_{\text {fuoco, }}$, anche proietterebbe il suo fuoco sul complemento "Shakespeare", evocando così un diverso insieme di elementi in gioco, dove il paradigma di alternative al fuoco dell'avverbio sarebbe dato non dalle "persone che leggono Shakespeare" bensì dagli "autori letti da Stella". Questo discorso, naturalmente, non si applica 
al parlato, dove intervengono, fra gli altri, fattori di natura prosodica a segnalare il dominio di associazione pertinente. In breve, l'ambiguità referenziale innescata dall'ampia mobilità distribuzionale di también deve essere risolta in modo da veicolare la stessa relazione e proiettare lo stesso insieme di elementi in gioco nella traduzione in italiano. Risulta necessario, in tal senso, identificare il fuoco dell'avverbio mediante la ricostruzione dello schema comparativo soggiacente, formato dal (i) termine presupposto che fa da sfondo comune ai (ii) termini comparati: (ii.a) fuoco dell'avverbio e (ii.b) paradigma di alternative. Gli avverbi della lingua italiana, infatti, hanno una configurazione più locale che richiede lo scioglimento dell'ambiguità superficiale, ammessa invece dalla lingua spagnola.

Il livello di articolazione gerarchico-informativa degli enunciati proposto dal MB consente, invece, di orientarsi nella polifunzionalità di también rispetto agli usi come avverbio focalizzante $v s$ connettivo testuale. Gli esempi analizzati dimostrano che, in effetti, esiste una correlazione tra la manifestazione (saturandola o con altri elementi lingusitici) nell'UI (Nucleo, Quadro o Appendice) in cui è collocato también e la specifica funzione discorsiva svolta, confermando, in tal modo, le osservazioni di Borreguero (2014) e De Cesare / Borreguero (2014). Le diverse soluzioni traduttive per la resa in italiano di también rispondono, infatti, soltanto in parte a scelte stilistiche della traduttrice. Nella maggior parte dei casi, invece, la selezione dei diversi traducenti è spiegabile sulla base del $\mathrm{MB}$. Da ciò deriva la grande produttività di questo modello come strumento euristico e descrittivo per l'analisi delle particelle dotate di polifunzionalità paradigmatica, anche in una prospettiva contrastiva.

Nello specifico, l'occorrenza di también entro l'unità nucleare attiva la funzione di avverbio focalizzante a discapito di quella connettiva, da cui deriva l'accettabilità degli equivalenti anche e pure in italiano. Il medesimo valore di focalizzatore è attivato dall'occorrenza di también nel Quadro Informativo insieme ad altri elementi linguistici che costituiscono, del tutto o in parte, il fuoco dell'avverbio. Diversamente, quando también satura senza residui il Quadro, a prevalere è la funzione di connettivo testuale. Di conseguenza, il traducente anche risulta inappropriato. In tal caso, occorre quindi selezionare altre forme dell'italiano, come e poi, inoltre, in più ecc., per veicolare la connessione additiva sul piano testuale. Si registra altresì che l'occorrenza di también come connettivo è marginale rispetto a quella di avverbio focalizzante. La ragione è da ascrivere probabilmente alla presenza di competitori linguistici specializzati nella connessione additiva tra enunciati, come, ad esempio, además, asimismo ed es más. O ancora, la marginalità di también come connettivo testuale si può imputare al suo processo di pragmaticalizzazione ancora in corso.

Per quanto riguarda l'occorrenza di también in Appendice, il discrimine per l'attribuzione della funzione di focalizzatore oppure di connettivo è - come nel caso del Quadro - dato dall'eventuale co-occorrenza di altri elementi linguistici di carattere denotativo. Più precisamente, quando también accompagna altri elementi linguistici, tende a prevalere la funzione di avverbio focalizzante, da cui deriva l'accettabilità del traducente anche. Diversamente, quando también satura l'Appendice, a essere attivata è la funzione connettiva che, in un'ottica interlinguistica, si traduce nella selezione di equivalenti come inoltre, in più, in aggiunta ecc., ma non di anche, analogamente a quanto osservato per il Quadro. Tuttavia, esistono delle eccezioni o, quanto meno, dei casi ambigui. Nonostante la saturazione dell'Appendice, infatti, fattori quali la prossimità al verbo principale e, quindi, al Nucleo Informativo possono favorire un'interpretazione di también come focalizzatore del sintagma verbale. 
In definitiva, il MB si è dimostrato utile per orientarsi nella polifunzionalità (avverbio focalizzante $v s$. connettivo testuale) delle particelle discorsive oggetto di analisi. D'altra parte, per via della configurazione più locale dell'italiano (vs spagnolo), è stato necessario inserire altri criteri di analisi, come l'identificazione della struttura comparativa proiettata dal focalizzatore. Ricostruire i termini comparati e il termine presupposto diventa cruciale nei casi in cui - indipendentemente dal valore semantico e dall'occorrenza nelle UI - il fuoco di también è posto, linearmente o a distanza, a sinistra dell'avverbio. In casi del genere, nella resa in italiano è necessaria la ripresa pronominale dopo gli avverbi anche e pure o, in alternativa, la riorganizzazione sintagmatica dell'enunciato, col fine di proiettare il medesimo insieme di elementi in gioco del testo originale.

\section{Riferimenti bibliografici}

Andorno, Cecilia (2000): Focalizzatori fra connessione e messa a fuoco. Il punto di vista delle varietà di apprendimento, Milano, Franco Angeli.

Andorno, Cecilia / De Cesare, Anna-Maria (2017): «Mapping additivity through translation. From French aussi to Italian anche and back in the Europarl-direct corpus», in Anna-Maria De Cesare, Cecilia Andorno (a c. di), Focus on Additivity. Adverbial modifiers in Romance, Germanic and Slavic languages, Amsterdam, John Benjamins, pp. 157-200.

Baker, Mona (1995): «Corpora in Translation Studies. An overview and suggestions for future research», Target, 7/2, pp. 223-243.

Bazzanella, Carla (2001): «Segnali discorsivi e contesto», in Wilma Heinrich, Christine Heiss (a c. di), Modalità e Substandard. International Conference Proceedings, Bologna, CLUEB, pp. 41-64.

Berrendonner, Alain (a c. di) (2012): Grammaire de la période, Berne, Peter Lang.

Borreguero Zuloaga, Margarita (2011): «Focalizzatori a confronto: anche vs. también», Studi italiani di linguistica teorica ed applicata, 40/3, pp. 441-468.

Borreguero Zuloaga, Margarita (2014): «Focalizadores aditivos escalares y posición enunciativa. Un estudio contrastivo español / italiano», Philologia Hispalensis, 28/3-4, pp. 13-57.

Borzi, Claudia (2009): «La expresión de la causa en la prensa escrita de Buenos Aires: el uso de ya que», Anales del Instituto de Lingüistica, 30-31, pp. 71-101.

Briz Gómez, Antonio / Grupo Val.Es.Co. (2003): «Un sistema de unidades para el estudio del lenguaje coloquial», Oralia, 6, pp. 7-61.

Briz Gómez, Antonio (1998 [2001]): El español coloquial en la conversación: esbozo de pragmagramática, Madrid, Arco/Libros.

Briz Gómez, Antonio / Pons Bordería, Salvador / Portolés Lázaro, José (a c. di) (2008): Diccionario de partículas discursivas del español. $<$ http://www.dpde.es $>$

Calvi, Maria Vittoria (2016): «La alternancia anche / también en el español hablado por inmigrantes hispanoamericanos en Italia», in María Eugenia Sainz González et al. (a c. di), Geométrica explosión: Estudios de lengua y literatura en homenaje a René Lenarduzzi, Venezia, Edizioni Ca' Foscari, pp. 147-160.

Carranza, Isolda (1998): Conversación y deixis del discurso, Córdoba, Publicaciones de la Universidad Nacional de Córdoba.

Covarrubias Horozco, Sebastián (1611 [2006]): Tesoro de la lengua castellana o española, a c. di Ignacio Arellano, Rafael Zafra, Madrid, Editorial Iberoamericana / Vervuert. 
Cuartero Sánchez, Juan Manuel (2002): Conectores y conexión aditiva. Los signos incluso, también y además en español actual, Madrid, Gredos.

De Cesare, Anna-Maria (2004): «L'avverbio anche e il rilievo informativo del testo», in Angela Ferrari (a c. di), La lingua nel testo, il testo nella lingua, Torino, Istituto dell'Atlante Linguistico Italiano, pp. 191-218.

De Cesare, Anna-Maria (2004a): «Y a-t-il encore quelque chose à ajouter sur l'italien anche? Une réponse basée sur le CORIS/CODIS», Rivista di Linguistica / Italian Journal of Linguistics, 16/1, pp. 3-34.

De Cesare, Anna-Maria (2010): «On the focusing function of focusing adverbs: A discussion based on Italian data», Linguistik online, 44/4, pp. 99-116.

De Cesare, Anna-Maria (2015): «Additive focus adverbs in canonical word order: A corpus-based study of It. anche, Fr. aussi and E. also in written news», Linguistik online, 71/2, pp. 31-56.

De Cesare, Anna-Maria (2019): «Avverbi focalizzanti additivi prototipici in tre lingue romanze: italiano, francese e rumantsch grischum a confronto», in Óscar Loureda, Martha Rudka, Giovanni Parodi (a c. di), Marcadores del discurso y lingüistica contrastiva en las lenguas románicas. Madrid, Iberoamericana / Vervuert, pp. 163-178.

De Cesare, Anna-Maria (2019a): Le parti invariabili del discorso, Roma, Carocci.

De Cesare, Anna-Maria / Borreguero Zuloaga, Margarita (2014): «The contribution of the Basel Model to the description of polyfunctional discourse markers: The case of It. anche, Fr. aussi, and Sp. también», in Salvador Pons Bordería (a c. di), Discourse Segmentation in Romance Languages, Amsterdam, John Benjamins, pp. 55-90.

Degand, Liesbeth / Simon, Anne Catherine (2009): «Mapping prosody and syntax as discourse strategies: How Basic Discourse Units vary across genres», in Anne Wichmann, Nicole Dehé, Dagmar Barth-Weingarten (a c. di), When Prosody Meets Pragmatics: Research at the Interface, Bingley, Emerald.

Esposito, Giorgia (2019): «La literatura ancilar después de Borges», in Veronica Orazi, Federica Cappelli, Iole Scamuzzi, Barbara Greco (a c. di), Trayectorias literarias hispánicas: tradición, innovación y nuevos paradigmas, Città, AISPI Edizioni, "Biblioteca AISPI", pp. 245-256.

Ferrari, Angela (2004): «La lingua nel testo, il testo nella lingua», in Angela Ferrari (a c. di), La lingua nel testo, il testo nella lingua, Torino, Istituto dell'Atlante Linguistico Italiano, pp. 9-41.

Ferrari, Angela et al. (2008): L'interfaccia lingua-testo: natura e funzioni dell'articolazione informativa dell'enunciato, Alessandria, Edizioni dell'Orso.

Ferrari, Angela et al. (2018): La punteggiatura italiana contemporanea. Un'analisi comunicativo-testuale, Roma, Carocci.

Fischer, Kerstin (2006): «Frames, constructions, and invariant meanings: The functional polysemy of discourse particles», in Kerstin Fischer (a c. di), Approaches to Discourse Particles, Amsterdam, Elsevier, pp. 427-447.

Fuentes Rodríguez, Catalina (1987): Enlaces extraoracionales, Sevilla, Alfar.

Fuentes Rodríguez, Catalina (2009): Diccionario de conectores y operadores del español, Madrid, Arco/Libros.

GDLE = Bosque, Ignacio / Demonte, Violeta (a c. di) (1999): Gramática descriptiva de la lengua española, vol. III, Madrid, Espasa Calpe.

Hartmann, Reinhard R. K. (2004): «Lexicography and translation», in Chan Sin-Wai (a c. di), Translation and Bilingual Dictionaries, Tübingen, Max Niemeyer Verlag, pp. 7-21. 
Holgado Lage, Anais (2017): Diccionario de marcadores discursivos para estudiantes de español como segunda lengua, New York, Peter Lang.

Kovacci, Ofelia (1999): «El adverbio», in Ignacio Bosque, Violeta Demonte (a c. di), Gramática descriptiva de la lengua española: sintaxis básica de las clases de palabras, Madrid, Espasa Calpe, vol. I, pp. 705-786.

Krifka, Manfred (2006 [2007]): «Basic notions of information structure», in Caroline Féry, Gisbert Fanselow, Manfred Krifka (a c. di), The Notions of Information Structure: Interdisciplinary Studies on Information Structure, Potsdam, Universitätsverlag Potsdam, vol. VI, pp. 13-55.

Lala, Letizia (2015): «Puntuación y reglas. La trattazione della punteggiatura nella normativa sulla lingua spagnola», RiCognizioni, 2/4, pp. 63-81.

Lombardi Vallauri, Edoardo / Tamburini, Fabio (2012): «Topic and Focus marking in an Italian corpus: Some results of algorithmic measurement and structural interpretation», in Heliana Mello, Massimo Pettorino, Tommaso Raso (a c. di), Proceedings of the VII GSCP International Conference: Speech and Corpora, Firenze, Firenze University Press, pp. 191-196.

Loureda, Óscar / Acín, Esperanza (a c. di) (2010): Los estudios sobre marcadores del discurso en español, hoy, Madrid, Arco/Libros.

Loureda, Óscar / Cruz, Adriana / Grupo Diskurspartikeln und Kognition (2013): «Aproximación experimental sobre los costes de procesamiento de las partículas focales del español también e incluso», Cuadernos AISPI, 2, pp. 75-98.

Martín Zorraquino, María Antonia / Montolío Durán, Estrella (a c. di) (1998 [2008]): Los marcadores del discurso: teoría y análisis, Madrid, Arco/Libros.

Martín Zorraquino, María Antonia / Portolés Lázaro, José (1999): «Los marcadores del discurso», in Ignacio Bosque, Violeta Demonte (a c. di), Gramática descriptiva de la lengua española: entre la oración y el discurso, Madrid, Espasa Calpe, vol. III, pp. 4051-4203.

Montolío Durán, Estrella (2001): Conectores de la lengua escrita: contraargumentativos, consecutivos, aditivos y organizadores de la información, Barcelona, Ariel.

Morel, Mary-Annick (2010): «Structure coénonciative du texte oral dialogué: intonation, syntaxe, regard et geste», in Ligia Stela Florea et al. (a c. di), Directions actuelles en linguistique du texte, Cluj-Napoca, Casa Cărţii de Ştiinţă, pp. 9-22.

Nadal, Laura / Cruz, Adriana / Recio, Inés / Loureda, Óscar (2016): «El significado procedimental y las partículas discursivas del español: Una aproximación experimental», Revista Signos, 49/1, pp. 52-77.

NGLE = Nueva gramática de la lengua española. Vol. II Sintaxis, Madrid, Espasa, 2009.

Nølke, Henning (1983): Les adverbes paradigmatisants: fonction et analyse. Revue Romane, 23.

Perrin-Naffakh, Anne-Marie (1996): «Aussi adjonctif: de la syntaxe à la sémantique», Le Français Moderne, 64/2, pp. 136-154.

Pons Bordería, Salvador (1998): Conexión y conectores: estudio de su relación en el registro informal de la lengua, Valencia, Universitat de Valencia.

Pons Bordería, Salvador (a c. di) (2014): Discourse Segmentation in Romance Languages, Amsterdam, John Benjamins.

Portolés Lázaro, José (1998 [2001]): Marcadores del discurso, Barcelona, Ariel.

Portolés Lázaro, José (2007): «Escalas informativas aditivas: pruebas del español», Spanish in Context, 4/2, pp. 135-157.

Portolés Lázaro, José (2010): «Los marcadores del discurso y la estructura informativa», in Óscar Loureda Lamas, Esperanza Acín Villa (a c. di), Los estudios sobre marcadores del discurso en español, hoy, Madrid, Arco/Libros, pp. 281-326. 
Reyes, Alfonso (1944): El deslinde. Prolegómenos a la Teoría literaria, México, El Colegio de México.

Reyes, Alfonso (1962): Obras completas, vol. XIV, México, Fondo de Cultura Económica.

Ricca, Davide (2017): «Meaning both 'also' and 'only'? The intriguing polysemy of Old Italian pur(e)», in Anna-Maria De Cesare, Cecilia Andorno (a c. di), Focus on Additivity. Adverbial Modifiers in Romance, Germanic and Slavic languages, Amsterdam, John Benjamins Publishing Company, pp. 45-76.

Roulet, Eddy / Filliettaz, Laurent / Grobet, Anne / Burger, Marcel (2001): Un modèle et un instrument d'analyse de l'organisation du discours, Berne, Peter Lang.

Sainz González, María Eugenia (2006): «También / anche: un estudio semántico contrastivo», in Gloria Bazzocchi, Pilar Capanaga (a c. di), Mediación lingüística de lenguas afines: español / italiano, Bologna, Gedit, pp. 23-45.

Sainz González, María Eugenia (2012): «Tra l'altro: conexión y focalización. Análisis contrastivo con el español», Cuadernos de filología italiana, 19, pp. 41-68.

Santos Río, Luis (2003): Diccionario de partículas, Salamanca, Luso-Española de Ediciones. Valdés, Juan (1736 [1987]): Diálogo de la lengua, a c. di Cristina Barbolani, Madrid, Cátedra. Vila-Matas, Enrique (2002): El mal de Montano, Barcelona, Anagrama. [Tr. it. di Natalia Cancellieri Il mal di Montano, Milano, Feltrinelli, 2005].

Visconti, Jacqueline (2005): «On the origins of scalar particles in Italian», Journal of Historical Pragmatics, 6/2, pp. 237-261. 